\title{
Prognostic value of the Glasgow prognostic score in colorectal cancer: a meta-analysis of 9,839 patients
}

This article was published in the following Dove Medical Press journal: Cancer Management and Research

\author{
Xin $\mathrm{Lu}^{\prime}$ \\ Wanying Guo ${ }^{2}$ \\ Wei $\mathrm{Xu}{ }^{\prime}$ \\ Xuelei Zhang' \\ Zhijie Shi' \\ Leizhen Zheng' \\ Wenzhao Zhao' \\ 'Department of Gastrointestinal \\ Surgery, The First Affiliated Hospital \\ and College of Clinical Medicine of \\ Henan University of Science and \\ Technology, Luoyang 471003, China; \\ ${ }^{2}$ Department of Breast Surgery, The \\ First Affiliated Hospital and College of \\ Clinical Medicine of Henan University \\ of Science and Technology, Luoyang \\ 47I003, China
}

Correspondence: Wenzhao Zhao Department of Gastrointestinal Surgery, The First Affiliated Hospital and College of Clinical Medicine of Henan University of Science and Technology, No. 636 Guanlin Road, Luoyang 471003, China $\mathrm{Tel} / \mathrm{fax}+8637969823275$ Email zhaowenzhaol23@foxmail.com
Purpose: The aim of this study was to perform a systematic review and meta-analysis to evaluate the value of the Glasgow prognostic score (GPS) or modified Glasgow prognostic score (mGPS) in patients with colorectal cancer (CRC).

Methods: A comprehensive medical literature search was performed using the online databases PubMed, Embase, Web of Science, and the Cochrane Library. After extracting basic characteristics and prognostic data from the included studies, overall survival (OS) and cancer-specific survival (CSS) were pooled as primary outcomes. Subgroup analyses were performed according to therapeutic strategies, models, cutoff values, regions, tumor, node, metastasis stages, sample size, and ages.

Results: Forty-three independent cohorts from 41 studies with 9,839 CRC patients were included in the present study. Correlation between GPS or mGPS and OS was analyzed in 32 cohorts of 7,714 patients, and 23 independent cohorts of 5,375 patients focused on the correlation between GPS or mGPS and CSS. The overall outcomes showed that patients with elevated GPS or mGPS were associated with poor OS (HR: $2.20,95 \%$ CI: $1.88-2.57, P<0.001)$. Elevated GPS or mGPS also resulted in worse CSS (HR: 1.86, 95\% CI: 1.59-2.17, $P<0.001$ ). The results of the subgroup analyses confirmed the overall outcomes.

Conclusion: GPS or mGPS is an accurate prognostic predictor in patients with CRC. Patients with elevated pretreatment GPS or mGPS have a poor prognosis. Subgroup analyses confirmed the overall outcomes. Pretreatment GPS is a useful biomarker in the management of CRC

Keywords: colorectal cancer, Glasgow prognostic score, modified Glasgow prognostic score, systematic review, meta-analysis

\section{Introduction}

Colorectal cancer (CRC) is one of the most common gastrointestinal malignancies and the fourth leading cause of cancer-related mortality worldwide. ${ }^{1,2} \mathrm{CRC}$ accounts for $\sim 10 \%$ of all newly diagnosed cancers each year. ${ }^{3}$ Although diagnostic technologies and therapeutic strategies for CRC have markedly improved, the prognosis of patients remains poor, which is attributed to the high rate of tumor recurrence and metastasis. ${ }^{2,4}$ The treatment strategies for CRC are based on the biological characteristics of the tumor and the systemic condition of patients. Surgery remains the optimal curative treatment for resectable cancer and the optional strategy for many patients with advanced cancer, ${ }^{5}$ while chemotherapy, immunotherapeutic strategies, and targeted therapy are optional for unresectable cancers. ${ }^{4,5}$ Therefore, an accurate prediction model which can predict the prognosis of CRC patients would be useful for the selection of therapeutic modalities. 
In previous studies, the tumor, node, metastasis (TNM) stage, which was proposed by the American Joint Committee on Cancer, was considered an effective system for predicting CRC recurrence and patient prognosis. ${ }^{6,7}$ However, the TNM stage alone was demonstrated to be inadequate in evaluating prognostic outcomes as tumor progression may be determined by the tumor characteristics as well as systemic inflammation and nutritional status. ${ }^{8,9}$ Recently, an increasing number of studies have focused on the prognostic role of inflammation biomarkers in predicting the prognosis of malignancies. ${ }^{10-12}$ The Glasgow prognostic score (GPS), an inflammationbased model, has been shown to be an accurate predictor of prognosis in CRC patients in several studies. ${ }^{13-17}$ This score is based on the combination of C-reactive protein (CRP) and serum albumin (ALB) levels. It was first reported by Forrest et al in 2003 for its prognostic value in non-small-cell lung cancer. ${ }^{18}$ GPS was defined based on the presence of hypoalbuminemia $(<35 \mathrm{~g} / \mathrm{L})$ and elevated CRP $(>10 \mathrm{mg} / \mathrm{L})$ : if both were abnormal, the score was 2 ; if either was abnormal, the score was 1 ; if neither was abnormal, the score was $0 .{ }^{19-21}$ Subsequently, more studies have evaluated the prognostic role of the GPS in a variety of cancers, such as pancreatic cancer, esophageal cancer, and hepatocellular carcinoma. ${ }^{12,22,23}$ In addition, some centers applied the modified Glasgow Prognostic Score (mGPS) to evaluate the prognostic outcomes of CRC patients. mGPS is also calculated using CRP and ALB levels. Patients with $\mathrm{CRP}<10 \mathrm{mg} / \mathrm{L}$ were scored 0 , those with $\mathrm{CRP}>10 \mathrm{mg} / \mathrm{L}$ were scored 1 , and those with CRP $>10 \mathrm{mg} / \mathrm{L}$ and ALB $<35 \mathrm{~g} / \mathrm{L}$ were scored $2 .{ }^{20}$ However, the role of the GPS in CRC remains controversial. Ishizuka et al verified the accurate predictive value of GPS, ${ }^{24}$ while other researchers showed no association between the GPS and the prognosis of CRC patients. ${ }^{13,19,25,26}$

In this study, we investigated the prognostic value of pretreatment GPS in CRC patients by searching available relevant studies and conducting a meta-analysis. We also investigated the predictive role of the GPS in patients in different subgroups by subgroup analysis.

\section{Methods}

\section{Literature search strategy}

A comprehensive medical literature search was performed in May 2018 using the online databases PubMed, Embase, Web of Science, and the Cochrane Library. Studies which focused on the association between GPS and the prognostic outcomes of CRC patients were retrieved. There were no restrictions on language, publication region, and type. Search terms were confined to the following freetext words and Medical Subject Headings: ((C-reactive protein) or (CRP) or (albumin) or (Glasgow prognostic score) or (GPS)) and ((colorectal) or (colon) or (rectum) or (rectal)) and ((cancer) or (cancers) or (tumor) or (tumors) or (carcinoma)). A backward search was also conducted using cross-references from the bibliographies of primary selected studies and relevant studies to ensure a comprehensive search. Two reviewers ( $\mathrm{Lu}$ X and Guo WY) completed searching for titles and abstracts independently.

\section{Inclusion and exclusion criteria}

Two reviewers ( $\mathrm{Lu} \mathrm{X}$ and Guo WY) selected eligible studies independently based on the prespecified inclusion and exclusion criteria. When there were disagreements, a final decision was made by a senior reviewer (Zhao WZ). Inclusion and exclusion criteria were established by all authors.

The inclusion criteria were as follows: 1) studies evaluating patients with CRC; 2) studies evaluating either GPS or mGPS in patients prior to treatment; and 3) studies with a clear presentation of the main outcomes including overall survival (OS) and cancer-specific survival (CSS).

The exclusion criteria were as follows: 1) studies not focusing on the prognosis of CRC patients; 2) studies without survival data; 3 ) studies not focusing on either pretreatment GPS or mGPS; 4) review articles/editorials; and 5) conference abstracts/case reports.

For duplicate publications by the same authors or departments, only the publications with most representative patient cohorts were included in this meta-analysis. If two or more independent sample sets such as training cohorts and validation cohorts were analyzed in the same study, the cohorts were analyzed independently. Moreover, if the researches were repetitive, only one was included. A flow diagram of study retrieval and selection is shown in Figure 1.

\section{Data management and statistical analyses}

EndNote software (version X7, Thomson Reuters, USA) was used for sorting and preliminary screening. Data from the included studies were extracted by two authors (Xu W and Zhang XL) by reading the full text independently. Baseline information including the full list of authors, year of publication, regions of the research, research centers, sample size, follow-up period, TNM stages, and therapeutic strategies was summarized. The endpoints of OS and CSS were characterized by HRs with $95 \%$ CIs.

Data were extracted from tables or the text of the included studies. In some studies, the HRs and 95\% CIs were not presented in the tables or text. These values were then computed from the Kaplan-Meier graph using the Engauge Digitizer 


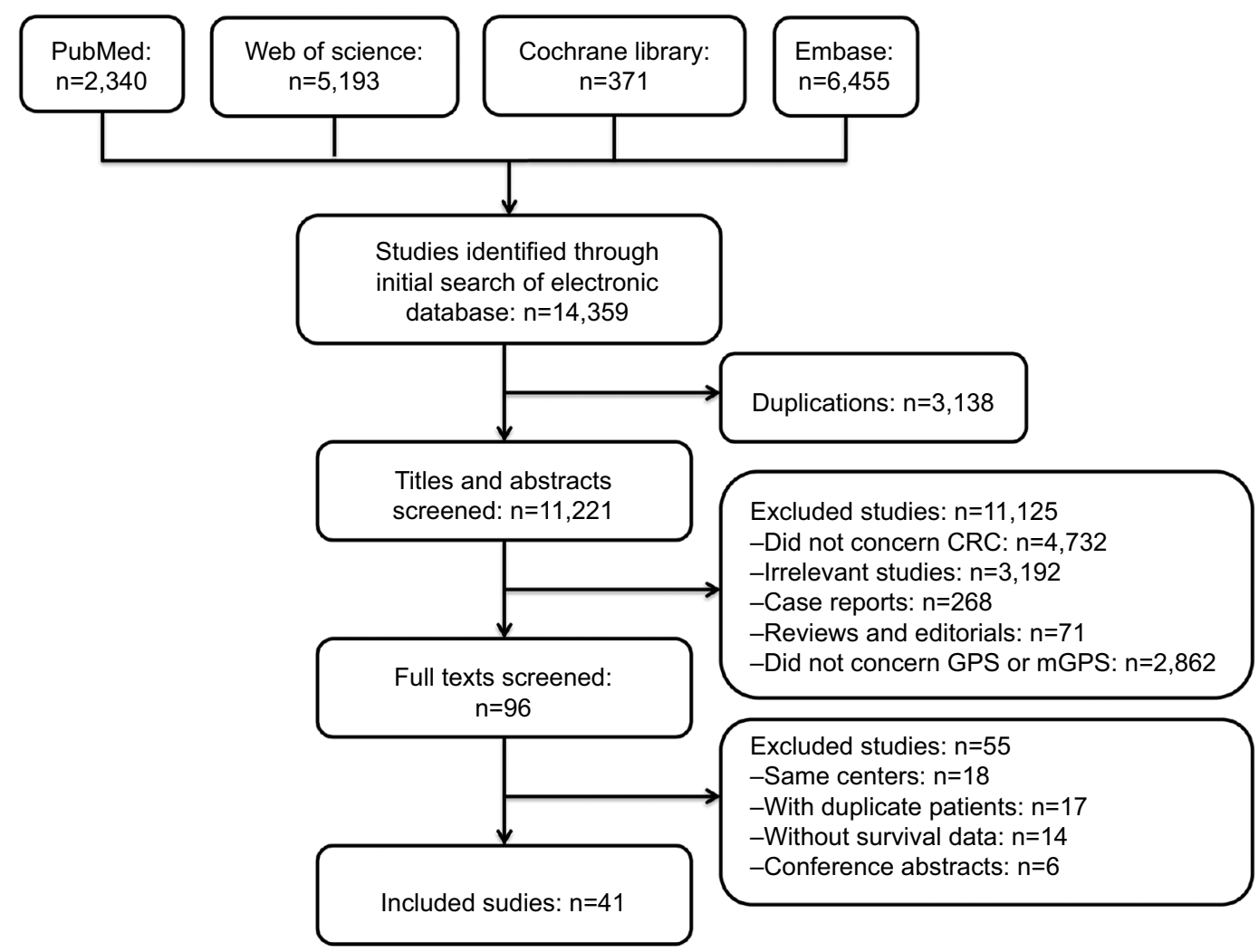

Figure I Flow diagram showing study retrieval and selection process.

Abbreviations: CRC, colorectal cancer; GPS, Glasgow prognostic score; mGPS, modified Glasgow prognostic score.

software (version 4.1, M Mitchell, Engauge Digitizer, http:// digitizer.sourceforge.net). ${ }^{27,28}$ All data from the included studies were pooled using the Cochrane Collaboration's Review Manager 5.3 (Cochrane Collaboration, Oxford, UK). A fixed effects model was used when there was no obvious heterogeneity $\left(\mathrm{I}^{2}=0\right)$; otherwise, a random effects model was used. Statistical heterogeneity among the studies was determined using the chi-squared test with a significance level of $P=0.10$, and heterogeneity was quantified using the $\mathrm{I}^{2}$ statistic. A sensitivity analysis of OS was performed using Stata software (version 12.0; StataCorp LP, College Station, TX, USA). ${ }^{29}$ Funnel plots were used to evaluate publication bias. Symmetry of the funnel plots was analyzed using Egger and Begg tests (Stata, version 12.0).

\section{Risk of bias assessment}

All the included studies were critically assessed for methodological quality by two researchers independently ( $\mathrm{Lu} \mathrm{X}$ and Guo WY) by using the Quality In Prognosis Studies tool. ${ }^{30}$ Each study was graded for the following domains: study participation, study attrition, prognostic factor measurement, outcome measurement, study confounding, and statistical analysis and reporting. The risk of bias for each domain is graded as low $(-)$, moderate $( \pm)$, or high $(+)$.

\section{Subgroup analyses}

Subgroup analyses were performed according to the models used to predict the prognostic outcomes of CRC patients as the scoring of the GPS and mGPS models was different. Subgroups were set according to therapeutic strategies, score cutoff values, sample size, region of publication, and TNM stages of patients in the included studies. The treatment modalities included surgical resection (SR) and chemotherapy. The cutoff value for sample size in the studies was a total of 300 patients. The region subgroups were defined as Asian countries and countries out of Asia taking into consideration the differences between the epidemiologic features and clinicopathological characteristics of CRC. Subgroups of TNM stages were divided into patients with advanced tumors of TNM stage IV and patients with TNM stage 0-III.

\section{Results}

\section{Characteristics of the included studies}

The comprehensive literature search identified a total of 14,359 studies from the above four databases. A flow diagram of study identification and selection is shown in Figure 1. Of these studies, 3,138 were duplicates. The titles and abstracts of the remaining 11,221 studies 
were then screened. Among the 11,125 studies excluded, 4,732 were not related to CRC, 3,192 were irrelevant studies, 268 were case reports, 71 were reviews or editorials, and 2,862 did not evaluate GPS or mGPS. The full texts of the remaining 96 articles were carefully reviewed, 56 articles were excluded, and 41 articles were finally included in the present study. Of the 56 excluded studies, 18 studies were published by the same center, 18 included duplicate sample sets, 14 did not include survival outcomes, and six were conference abstracts. Two studies were published by the same author in the same year. ${ }^{31}$ However, the patients enrolled in these studies received different treatment modalities (SR and chemotherapy). As a result, both studies were included in this meta-analysis. Two other studies were published by the same center ${ }^{13,24}$ and both were included as independent cohorts due to their different endpoints. Patient cohorts in these studies were analyzed independently. Hence, 43 independent cohorts from 41 studies were included in this study. ${ }^{13-17,19-21,24-26,31-59}$ The methodological quality of the included studies is summarized in Table S1.

The characteristics of the included studies are summarized in Table 1. A total of 9,839 patients were enrolled. Thirty-three independent cohorts of 8,006 CRC patients analyzed the correlation between GPS or mGPS and OS, and 26 independent cohorts of 7,616 patients focused on the correlation between GPS or mGPS and CSS. The overall outcomes showed that patients with elevated GPS or mGPS were associated with poor OS (HR: $2.20,95 \%$ CI: $1.88-2.57, P<0.001)$. In addition, higher GPS or mGPS resulted in worse CSS (HR: 1.86, 95\% CI: 1.59-2.17, $P<0.001)$.

\section{Subgroup analysis}

The results of the subgroup analyses are shown in Table 2 . In the model subgroups, elevated GPS resulted in worse OS (HR: $2.08,95 \%$ CI: $1.69-2.55, P<0.001)$ and CSS (HR: $2.38,95 \%$ CI: $1.63-3.46, P<0.001$ ). Patients in the mGPS subgroup with elevated mGPS were associated with a worse OS (HR: $2.23,95 \%$ CI: $1.79-2.78, P<0.001)$ and CSS (HR: $1.73,95 \%$ CI: 1.47-2.03, $P<0.001$ ) (Figure 2). Patients with increased GPS or mGPS who underwent SR had a poor OS (HR: $2.30,95 \%$ CI: $1.90-2.79, P<0.001$ ) and CSS (HR: $2.06,95 \% \mathrm{CI}: 1.67-2.53, P<0.001$ ). The chemotherapy subgroup confirmed that elevated GPS or mGPS was associated with a worse OS (HR: 1.95, 95\% CI: $1.46-2.62, P<0.001)$ and CSS (HR: $1.47,95 \% \mathrm{CI}$ : $1.24-1.74, P<0.001$ ) (Figure 3 ). The pooled outcomes of studies which used 1 as the cutoff value demonstrated that

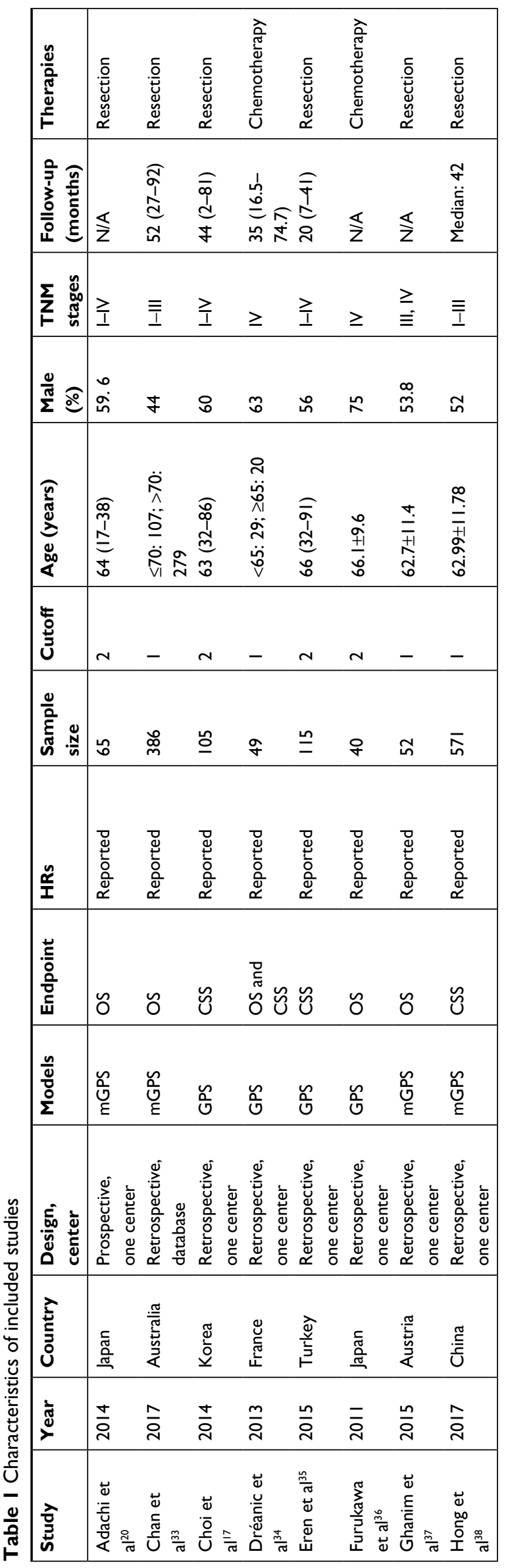




\begin{tabular}{|c|c|c|c|c|c|c|c|c|c|c|c|c|c|c|c|c|c|}
\hline 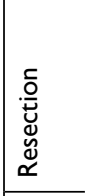 & 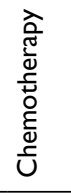 & 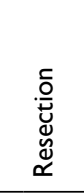 & 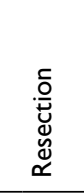 & 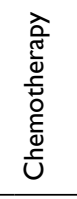 & 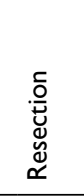 & 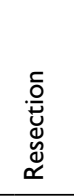 & 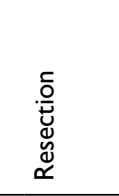 & 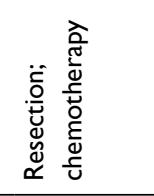 & 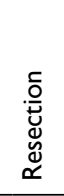 & 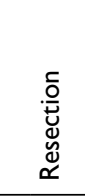 & 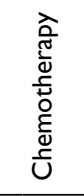 & $\begin{array}{l}\check{0} \\
\stackrel{\bar{\Xi}}{\mathscr{u}} \\
\propto \\
\propto\end{array}$ & 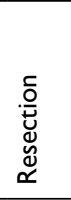 & 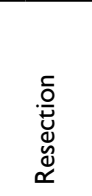 & 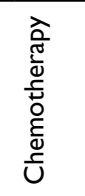 & 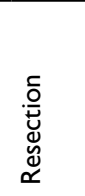 & 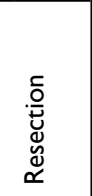 \\
\hline $\begin{array}{l}\frac{\sigma}{\alpha} \\
\frac{\infty}{d} \\
\text { ปn }\end{array}$ & $\frac{\frac{b}{+1}}{\frac{+1}{2}}$ & 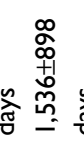 & 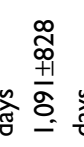 & 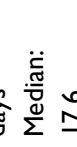 & 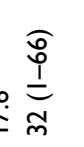 & 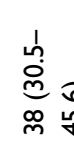 & 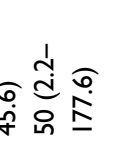 & 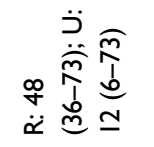 & 옹 & 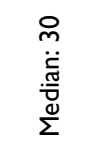 & 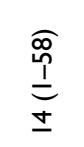 & 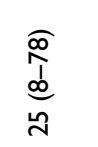 & & 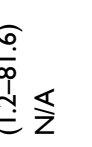 & $\begin{array}{l}6 \\
f \\
j \\
0 \\
\simeq\end{array}$ & 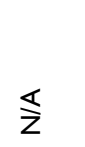 & $\begin{array}{l}\frac{\pi}{0} \\
\frac{n}{1} \\
\frac{n}{y} \\
m\end{array}$ \\
\hline$\overline{\bar{I}}$ & $\geq$ & 궁 & \a & $\geq$ & $\geq$ & $\geq$ & $\geq$ & $\begin{array}{l}\ddot{\overline{\bar{I}}} \geq \\
\ddot{\ddot{\alpha}}\end{array}$ & $=$ & $\geq$ & $\geq$ & $\bar{\equiv}$ & $\bar{\equiv}$ & $\geq$ & $\geq$ & $\stackrel{\geq}{I}$ & $\geq$ \\
\hline$\pi$ & 8 & 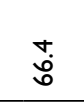 & $\stackrel{\infty}{\infty}$ & กิ & กై & 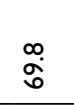 & $\begin{array}{l}\infty \\
\text { ஸे }\end{array}$ & 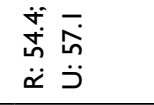 & 芦 & $\begin{array}{c}m \\
\stackrel{m}{n}\end{array}$ & mై & $\stackrel{m}{\stackrel{m}{n}}$ & 䓍 & $\stackrel{\infty}{\widetilde{\sigma}}$ & 華 & 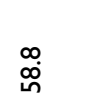 & $\hat{0}$ \\
\hline $\begin{array}{l}\widehat{N} \\
\infty \\
1 \\
m \\
0 \\
\pm \\
0\end{array}$ & 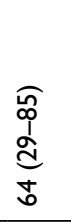 & 玄 & 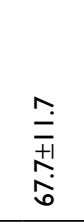 & 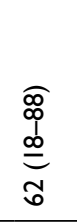 & $\begin{array}{l}\stackrel{0}{ } \\
\dddot{\rho} \\
\hat{\lambda} \\
\dot{q} \\
\ddot{\rho} \\
v 1\end{array}$ & 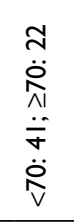 & 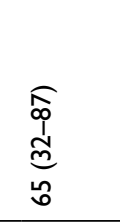 & 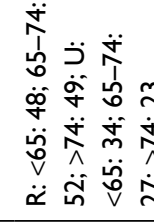 & $\begin{array}{l}2 \\
0 \\
0 \\
0 \\
0 \\
0 \\
0\end{array}$ & 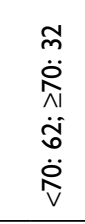 & 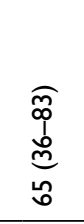 & 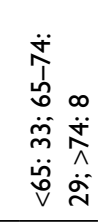 & $\begin{array}{l}\frac{0}{0} \\
\frac{0}{\alpha+1} \\
0 \\
0\end{array}$ & 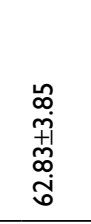 & 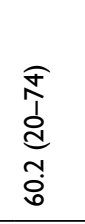 & 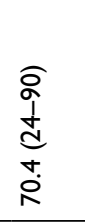 & 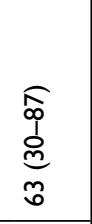 \\
\hline- & - & - & $\sim$ & $N$ & $N$ & - & - & N & $N$ & $N$ & $N$ & - & - & - & - & N & - \\
\hline$\underline{\underline{n}}$ & $\underline{\underline{0}}$ & $\bar{\lambda}$ & స్ & 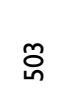 & $\stackrel{a}{R}$ & గె & $\underset{\sigma}{\sigma}$ & 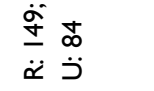 & ๙ & むু & $\infty$ & R & 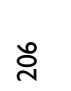 & $\stackrel{m}{m}$ & $\stackrel{\infty}{\square}$ & $\underset{\sim}{\mathbb{N}}$ & $\stackrel{+}{\underline{m}}$ \\
\hline 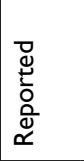 & 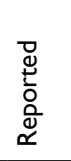 & 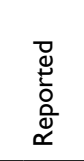 & 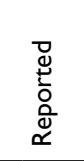 & 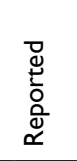 & 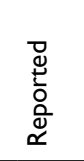 & 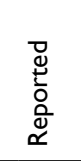 & 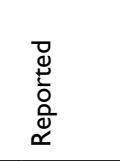 & 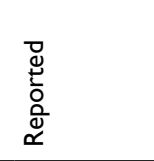 & 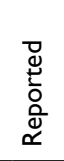 & 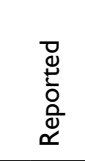 & 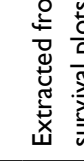 & 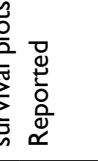 & 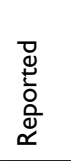 & 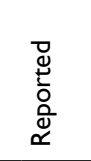 & 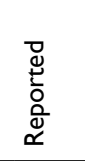 & 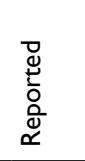 & 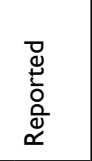 \\
\hline 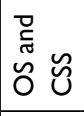 & & $\widetilde{U}$ & ő & 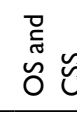 & & ő & ő & 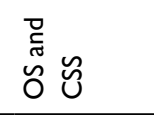 & ô & ŏ & 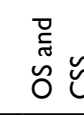 & $\hat{\beta} \tilde{u}$ & oิ & $\widetilde{U}$ & ŏ & oิ & 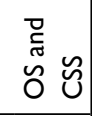 \\
\hline 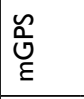 & $\begin{array}{l}\stackrel{n}{0} \\
\underline{0}\end{array}$ & 品 & on & $\stackrel{n}{\stackrel{n}{0}}$ & $\underset{\Xi}{\stackrel{n}{0}}$ & $\frac{n}{0}$ & 恕 & $\begin{array}{l}\stackrel{n}{0} \\
\underline{0} \\
\varepsilon\end{array}$ & on & 号 & â & $\stackrel{n}{0}$ & $\stackrel{n}{0}$ & 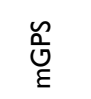 & : & 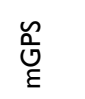 & 品 \\
\hline 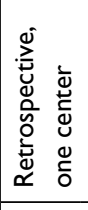 & 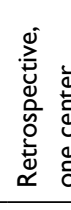 & 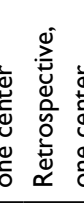 & 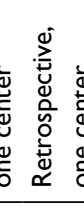 & 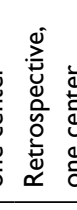 & 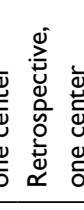 & 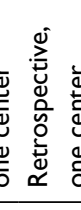 & 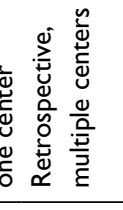 & 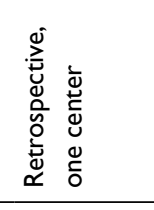 & 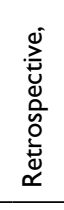 & 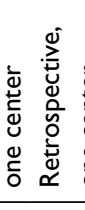 & 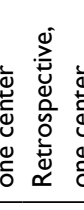 & 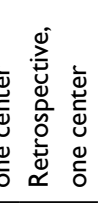 & 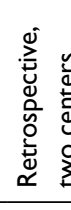 & 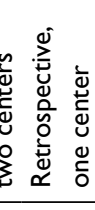 & 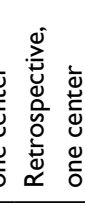 & 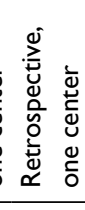 & 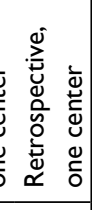 \\
\hline 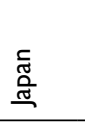 & 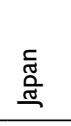 & $\begin{array}{l}\text { I } \\
\text { 兽 } \\
\end{array}$ & $\begin{array}{l}\text { I } \\
\text { I } \\
\end{array}$ & 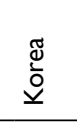 & 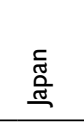 & 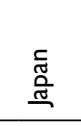 & 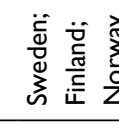 & J & 苞 & 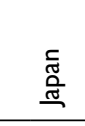 & 莺 & 弚 & 弚 & 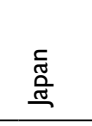 & 莺 & $\begin{array}{l}\text { 总 } \\
\text { 尊 } \\
\end{array}$ & 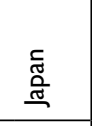 \\
\hline$\overline{\bar{N}}$ & $\frac{m}{i}$ & $\frac{\sim}{a}$ & $\frac{\circ}{2}$ & $\overline{\bar{\nu}}$ & $\frac{m}{a}$ & 음 & $\stackrel{\circ}{\circ}$ & ఫ્సે & $\frac{n}{a}$ & $\frac{m}{i}$ & $\frac{\pi}{a}$ & $\overline{\bar{N}}$ & $\frac{\circ}{2}$ & $\frac{ \pm}{\grave{N}}$ & $\frac{0}{\bar{N}}$ & $\frac{\nabla}{i}$ & $\overline{\bar{N}}$ \\
\hline 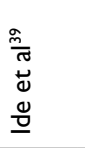 & 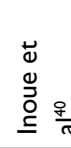 & 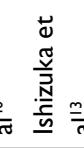 & 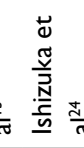 & 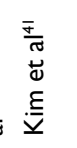 & 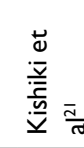 & 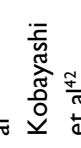 & 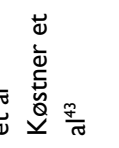 & 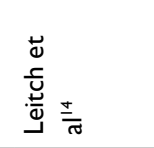 & 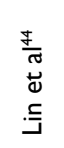 & 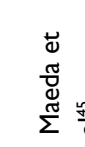 & 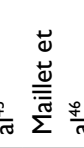 & 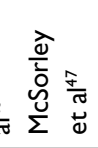 & 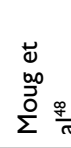 & 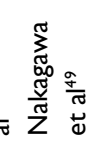 & 量 & 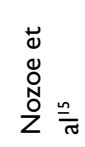 & 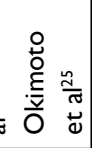 \\
\hline
\end{tabular}




\begin{tabular}{|c|c|c|c|c|c|c|c|c|c|c|c|c|c|c|c|c|}
\hline 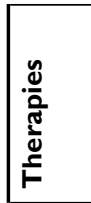 & 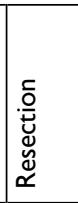 & 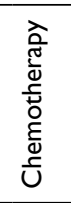 & 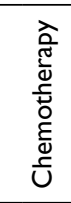 & 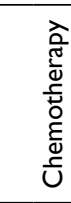 & 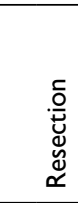 & 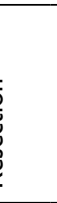 & 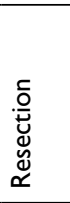 & 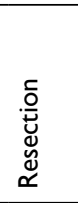 & 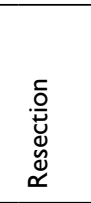 & 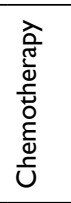 & 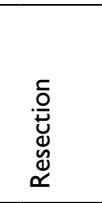 & 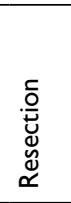 & 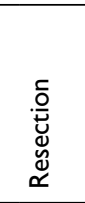 & 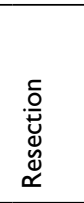 & 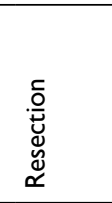 & 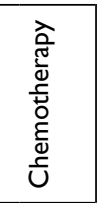 \\
\hline 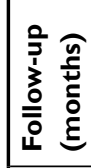 & 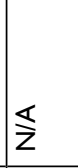 & 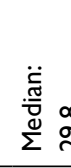 & & $\overleftarrow{z}$ & 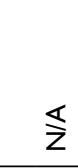 & & 공 & 苛 & 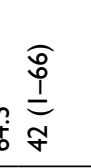 & $\bar{m} \frac{\hat{m}}{\tilde{m}}$ & 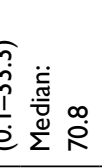 & 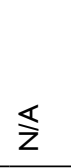 & 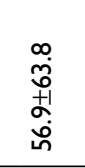 & 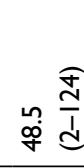 & 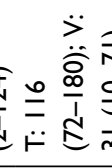 & 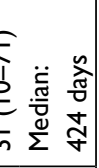 \\
\hline 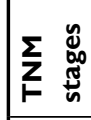 & I & $\geq$ & $\geq$ & $\geq$ & $\equiv$ & & $\geq$ & $\geqq$ & $\overline{\bar{I}}$ & $\geq$ & $\stackrel{\equiv}{\equiv}$ & $\bar{\equiv}$ & $\begin{array}{l}\equiv \\
\equiv\end{array}$ & $\underline{\equiv}$ & $\overline{\bar{o}}$ & $\geq$ \\
\hline 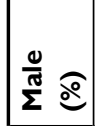 & 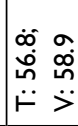 & & ఫे & $\stackrel{n}{n}$ & fं & & $\frac{\sigma}{\sigma}$ & ò & 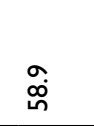 & $\begin{array}{l}\text { oे } \\
\text { oे }\end{array}$ & $\overline{i n}$ & ஸે & $\overline{\mathrm{S}}$ & กั & 访 & $\frac{\sigma}{\sigma}$ \\
\hline 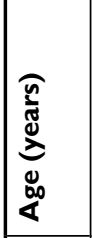 & 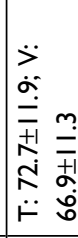 & $\begin{array}{l}\frac{a}{0} \\
\frac{1}{0} \\
\frac{1}{0}\end{array}$ & 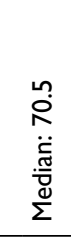 & 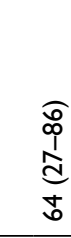 & 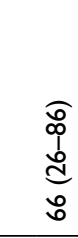 & 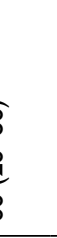 & $\begin{array}{l}\widehat{D} \\
\frac{1}{n} \\
0 \\
n \\
0\end{array}$ & 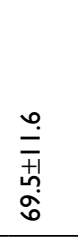 & 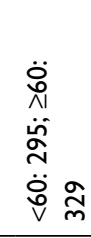 & 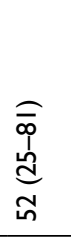 & 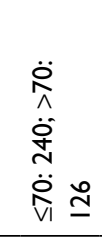 & 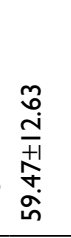 & $\begin{array}{l}\widehat{\bar{a}} \\
\stackrel{1}{d} \\
\stackrel{0}{0}\end{array}$ & $\begin{array}{l}\widehat{a} \\
\hat{\alpha} \\
\stackrel{0}{\varrho} \\
o \\
0\end{array}$ & 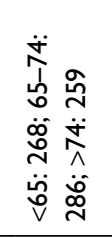 & 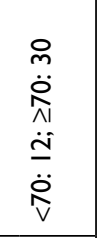 \\
\hline uั. & N & - & $N$ & - & - & & $\sim$ & - & $N$ & - & $N$ & - & - & - & - & $\sim$ \\
\hline 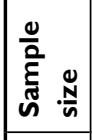 & 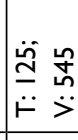 & 5 & กี & 으 & 岕 & & $\approx$ & $\bar{\lambda}$ & 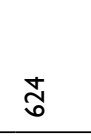 & $\underline{\Sigma}$ & : & $\stackrel{\text { 足 }}{\mathrm{N}}$ & $\frac{\sigma}{N}$ & $\begin{array}{l}\infty \\
o ̛ ⿱ \\
o\end{array}$ & $\frac{m}{\infty}$ & $\mathcal{F}$ \\
\hline$\stackrel{\check{\tilde{x}}}{\underline{\underline{I}}}$ & 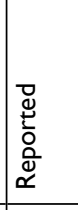 & 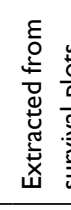 & 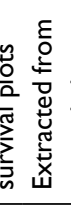 & 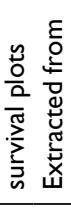 & 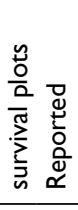 & & 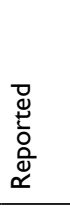 & 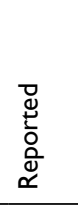 & 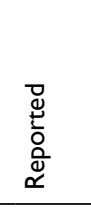 & 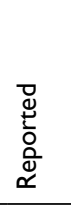 & 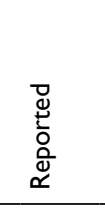 & $\begin{array}{l}\mathbb{\Xi} \\
\stackrel{ \pm}{0} \\
\stackrel{Q}{Q} \\
\propto \\
\end{array}$ & 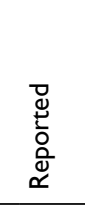 & 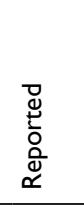 & 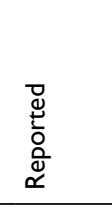 & 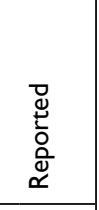 \\
\hline 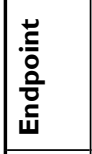 & ő & oิ & ő & $\begin{array}{l}\text { गे } \\
\text { लू } \\
\text { o }\end{array}$ & & & oั & 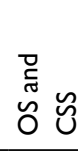 & & oั & $\tilde{U}$ & 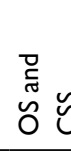 & 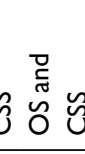 & 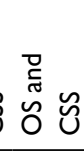 & 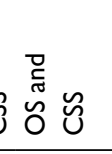 & $\widetilde{U}$ \\
\hline $\begin{array}{l}\frac{n}{0} \\
\frac{0}{0} \\
\frac{0}{\Sigma}\end{array}$ & 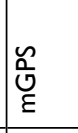 & ô & 苍 & 芩 & ô & & 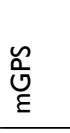 & 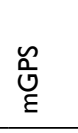 & $\begin{array}{l}\tilde{n} \\
0 \\
\underline{0}\end{array}$ & 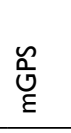 & ô & $\begin{array}{l}n \\
\text { ON } \\
\varepsilon\end{array}$ & 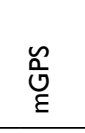 & $\begin{array}{l}\tilde{n} \\
0 \\
\underline{0} \\
\end{array}$ & 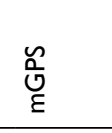 & $\begin{array}{l}\check{n} \\
\text { Uू } \\
\text { E }\end{array}$ \\
\hline 容 & 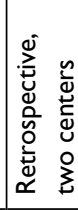 & 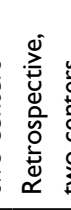 & 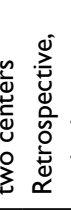 & 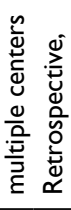 & 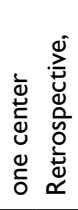 & 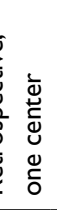 & 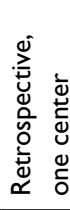 & 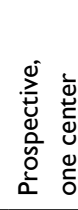 & 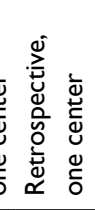 & 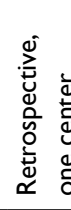 & 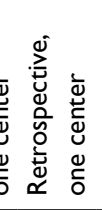 & 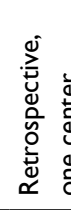 & 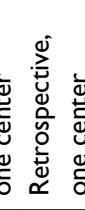 & 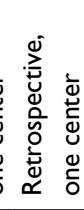 & 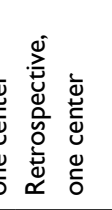 & 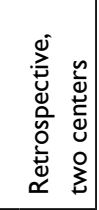 \\
\hline 胥 & $\begin{array}{l}\text { 总 } \\
\text { 兽 } \\
\end{array}$ & 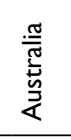 & 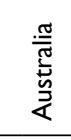 & $\begin{array}{l}\text { ్ㅠ } \\
\text { 尊 }\end{array}$ & $\begin{array}{l}\text { 。․ } \\
\text { 总 } \\
\end{array}$ & 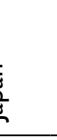 & $\begin{array}{l}\text { 总 } \\
\text { 首 } \\
\end{array}$ & 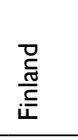 & $\begin{array}{l}\stackrel{\Xi}{0} \\
\stackrel{0}{0} \\
\end{array}$ & $\begin{array}{l}\stackrel{\Xi}{\Xi} \\
\stackrel{0}{0} \\
\underline{y}\end{array}$ & $\begin{array}{l}\text { 总 } \\
\text { 吾 } \\
\end{array}$ & $\begin{array}{l}\stackrel{\pi}{\tilde{E}} \\
\end{array}$ & $\begin{array}{l}\text { 总 } \\
\text { 尊 } \\
\end{array}$ & $\begin{array}{l}\text { 总 } \\
\text { 晋 } \\
\end{array}$ & 弚 & $\begin{array}{l}\text { Ĩ } \\
\text { 忢 } \\
\end{array}$ \\
\hline ঠ & $\frac{\infty}{2}$ & ઠั่ & ષ્̀ & 늠 & $\stackrel{n}{\circ}$ & ? & $\overline{\bar{N}}$ & $\frac{\infty}{2}$ & $\frac{m}{i}$ & ำ & $\stackrel{\text { กิ }}{\circ}$ & $\frac{\pi}{\circ}$ & $\overline{\bar{i}}$ & ํํำ & $\stackrel{\circ}{\circ}$ & $\frac{\text { ก }}{2}$ \\
\hline 흘 & 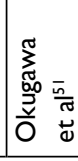 & $\begin{array}{l}\breve{\Delta} \\
\stackrel{\Xi}{\varpi}\end{array}$ & 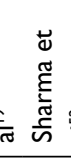 & 胥 & 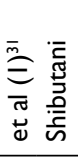 & 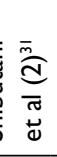 & 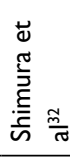 & 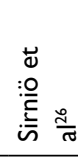 & 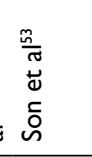 & 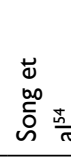 & 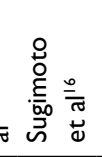 & $\begin{array}{l}\frac{n}{n} \\
\stackrel{n}{0} \\
5 \\
5\end{array}$ & 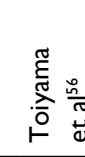 & 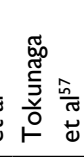 & 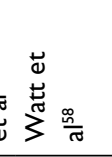 & 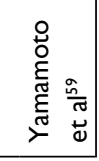 \\
\hline
\end{tabular}


Table 2 Results of subgroup analyses of overall survival and cancer-specific survival

\begin{tabular}{|c|c|c|c|c|c|c|c|c|}
\hline \multirow[t]{2}{*}{ Subgroups } & \multirow{2}{*}{$\begin{array}{l}\text { Independent } \\
\text { cohorts }\end{array}$} & \multirow{2}{*}{$\begin{array}{l}\text { Sample } \\
\text { size }\end{array}$} & \multirow{2}{*}{$\begin{array}{l}\text { HR (95\% Cl) } \\
(\mathrm{H} / \mathrm{L})\end{array}$} & \multirow[t]{2}{*}{$P$-value } & \multicolumn{4}{|c|}{ Study heterogeneity } \\
\hline & & & & & $\chi^{2}$ & df & $I^{2}(\%)$ & $P$-value \\
\hline Overall survival & 32 & $7,7 \mid 4$ & $2.20(1.88,2.57)$ & $<0.00001$ & 91.05 & 31 & 66 & $<0.00001$ \\
\hline \multicolumn{9}{|l|}{ Models } \\
\hline GPS & 14 & 2,293 & $2.08(1.69,2.55)$ & $<0.00001$ & 19.95 & 13 & 35 & 0.10 \\
\hline mGPS & 18 & 5,421 & $2.23(1.79,2.78)$ & $<0.00001$ & 70.68 & 17 & 76 & $<0.00001$ \\
\hline \multicolumn{9}{|l|}{ Therapies } \\
\hline Resection & 23 & 6,504 & $2.30(1.90,2.79)$ & $<0.0000$ I & 70.01 & 22 & 69 & $<0.00001$ \\
\hline Chemotherapy & 9 & 1,210 & $1.95(1.46,2.62)$ & $<0.00001$ & 19.36 & 8 & 59 & 0.01 \\
\hline \multicolumn{9}{|l|}{ Cutoff value } \\
\hline I & 18 & 4,263 & $1.85(1.58,2.16)$ & $<0.0000$ I & 37.66 & 17 & 55 & 0.003 \\
\hline 2 & 14 & 3,451 & $3.02(2.21,4.13)$ & $<0.0000$ I & 39.04 & 13 & 67 & 0.0002 \\
\hline \multicolumn{9}{|l|}{ Regions } \\
\hline Asia & 21 & 5,113 & $2.44(1.98,3.02)$ & $<0.00001$ & 56.26 & 20 & 64 & $<0.0001$ \\
\hline Others & 11 & 2,601 & $1.79(1.47,2.18)$ & $<0.00001$ & 20.44 & 10 & 51 & 0.03 \\
\hline \multicolumn{9}{|l|}{ Age, years } \\
\hline Mean/median $<65$ & 11 & 1,709 & $1.91(1.50,2.43)$ & $<0.00001$ & 21.98 & 10 & 55 & 0.02 \\
\hline Mean/median $\geq 65$ & 17 & 5,091 & $2.46(1.93,3.13)$ & $<0.00001$ & 67.21 & 16 & 76 & $<0.00001$ \\
\hline \multicolumn{9}{|l|}{ Sample size } \\
\hline Sample $<300$ & 24 & 3,256 & $2.43(1.97,3.00)$ & $<0.0000$ I & 60.13 & 23 & 62 & $<0.0001$ \\
\hline Sample $\geq 300$ & 8 & 4,458 & $1.86(1.50,2.31)$ & $<0.0000$ I & 21.04 & 7 & 67 & 0.004 \\
\hline \multicolumn{9}{|l|}{ TNM stages } \\
\hline $0-$ III & 12 & 3,588 & $1.95(1.57,2.42)$ & $<0.0000$ I & 31.86 & 10 & 69 & 0.0004 \\
\hline IV & 14 & 2,085 & $1.95(1.56,2.42)$ & $<0.0000$ I & 26.46 & 13 & 51 & 0.01 \\
\hline Cancer-specific survival & 23 & 5,375 & $1.86(1.59,2.17)$ & $<0.00001$ & 45.42 & 22 & 52 & 0.002 \\
\hline \multicolumn{9}{|l|}{ Models } \\
\hline GPS & 8 & 1,230 & $2.38(1.63,3.46)$ & $<0.00001$ & 12.75 & 7 & 45 & 0.08 \\
\hline mGPS & 15 & 4,145 & $1.73(1.47,2.03)$ & $<0.00001$ & 28.10 & 14 & 50 & 0.01 \\
\hline \multicolumn{9}{|l|}{ Therapies } \\
\hline Resection & 16 & 4,344 & $2.06(1.67,2.53)$ & $<0.0000$ I & 35.67 & 15 & 58 & 0.002 \\
\hline Chemotherapy & 7 & $|, 03|$ & $1.47(1.24,1.74)$ & $<0.00001$ & 6.06 & 6 & 1 & 0.42 \\
\hline \multicolumn{9}{|l|}{ Cutoff value } \\
\hline 1 & 14 & 3,852 & $1.74(1.48,2.05)$ & $<0.00001$ & 21.26 & 13 & 39 & 0.07 \\
\hline 2 & 9 & 1,523 & $2.34(1.61,3.40)$ & $<0.00001$ & 24.07 & 8 & 67 & 0.002 \\
\hline \multicolumn{9}{|l|}{ Regions } \\
\hline Asia & 15 & 3,744 & $2.01(1.65,2.44)$ & $<0.0000$ I & 27.86 & 14 & 50 & 0.01 \\
\hline Others & 8 & $|, 63|$ & $1.56(1.24,1.96)$ & $<0.00001$ & 10.65 & 7 & 34 & 0.15 \\
\hline \multicolumn{9}{|l|}{ Age, years } \\
\hline Mean/median $<65$ & 9 & 2,299 & $1.83(1.47,2.27)$ & $<0.0000$ I & 16.28 & 8 & 51 & 0.04 \\
\hline Mean/median $\geq 65$ & 10 & 2,512 & I.73 (I.34, 2.24) & $<0.0000$ I & 17.68 & 9 & 49 & 0.04 \\
\hline \multicolumn{9}{|l|}{ Sample size } \\
\hline Sample $<300$ & 17 & 2,311 & $2.02(1.64,2.49)$ & $<0.00001$ & 27.01 & 16 & 41 & 0.04 \\
\hline Sample $\geq 300$ & 6 & 3,064 & $1.61(1.32,1.96)$ & $<0.00001$ & 10.35 & 5 & 52 & 0.07 \\
\hline \multicolumn{9}{|l|}{ TNM stages } \\
\hline 0-III & 9 & 3,026 & $2.01(1.57,2.58)$ & $<0.00001$ & 19.29 & 8 & 59 & 0.01 \\
\hline IV & 10 & I,587 & $1.80(1.43,2.27)$ & $<0.0000$ I & 15.76 & 9 & 43 & 0.07 \\
\hline
\end{tabular}

Abbreviations: df, degrees of freedom; GPS, Glasgow prognostic score; H, high group; L, low group; mGPS, modified Glasgow prognostic score; TNM, tumor, node, metastases.

CRC patients with elevated GPS or mGPS had a worse OS (HR: $1.85,95 \%$ CI: $1.58-2.16, P<0.001$ ) and CSS (HR: $1.74,95 \%$ CI: $1.48-2.05, P<0.001)$. Studies which used 2 as the cutoff value also demonstrated that elevated GPS resulted in poor OS (HR: 3.02, 95\% CI: $2.21-4.13$, $P<0.001$ ) and CSS (HR: 2.34, 95\% CI: $1.61-3.40, P<0.001$ ) (Figure 4). The pooled outcomes of 21 independent cohorts published in Asia showed that increased GPS or mGPS was associated with worse OS (HR: 2.44, 95\% CI: 1.98-3.02, $P<0.001$ ) and CSS (HR: 2.01, 95\% CI: $1.65-2.44, P<0.001)$ (Figure 5). The subgroup analysis based on geographical regions showed that Asian patients with increased GPS or mGPS level had a poor OS (HR: $2.44,95 \%$ CI: $1.98-3.02$, $P<0.001)$ and CSS (HR: $2.01,95 \%$ CI: $1.65-2.44, P<0.001$ ) 
(Figure 6). For elderly patients with a mean/median age $>65$ years, elevated GPS or mGPS was also associated with worse OS (HR: 2.46, 95\% CI: 1.93-3.13, $P<0.001$ ) and CSS (HR: $1.73,95 \%$ CI: $1.34-2.24, P<0.001$ ) (Figure 7). The subgroup of patients with CRC TNM stage 0-III demonstrated the prognostic value of GPS or mGPS in predicting OS (HR: $2.13,95 \%$ CI: $1.66-2.73, P<0.001)$ and CSS (HR: 2.01, 95\% CI: 1.57-2.58, $P<0.001$ ) (Figure 8). The subgroup of patients with CRC TNM stage IV indicated that elevated GPS or mGPS was associated with poor OS (HR: $1.95,95 \%$ CI: $1.56-2.42, P<0.001)$ and CSS (HR: $1.80,95 \%$ CI: $1.43-2.27, P<0.001$ ).
A

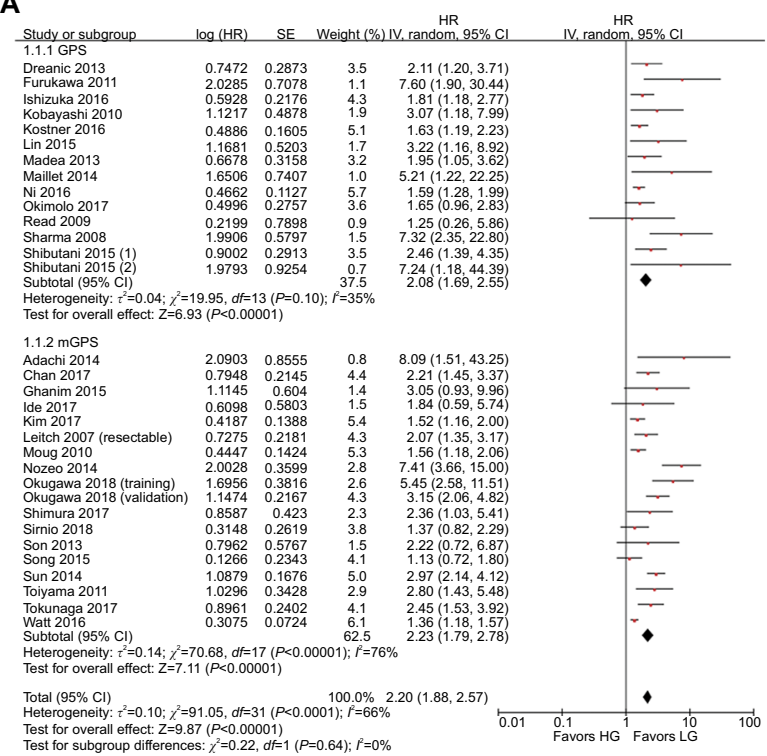

B

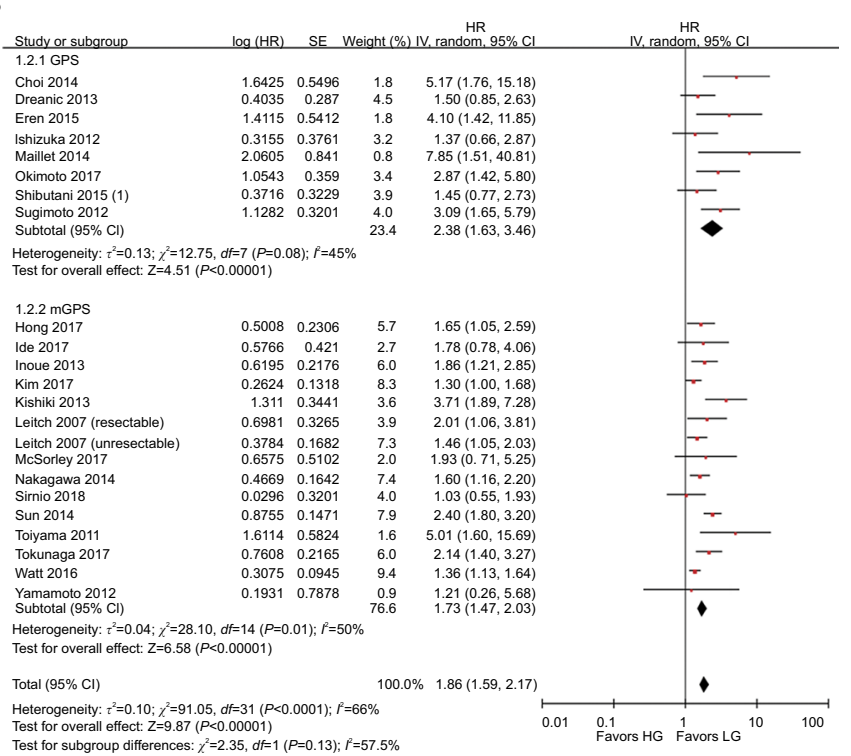

Figure 2 Subgroup analysis showing correlation between GPS and prognosis of CRC patients according to models.

Note: (A) Overall survival and (B) cancer-specific survival.

Abbreviations: CRC, colorectal cancer; df, degrees of freedom; GPS, Glasgow prognostic score; mGPS, modified Glasgow prognostic score; LG, low group; HG, high group; SE, standard error.

A

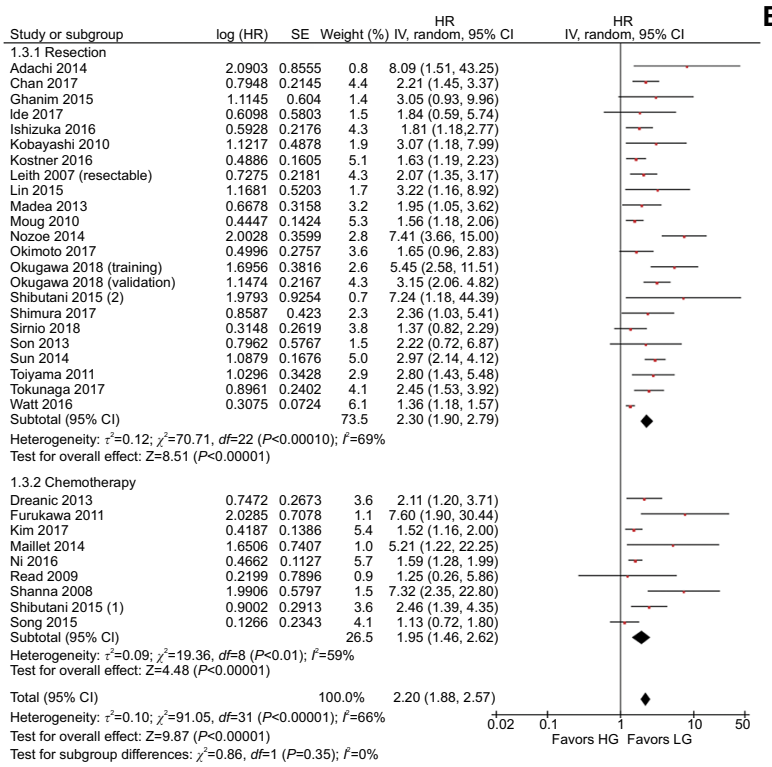

B

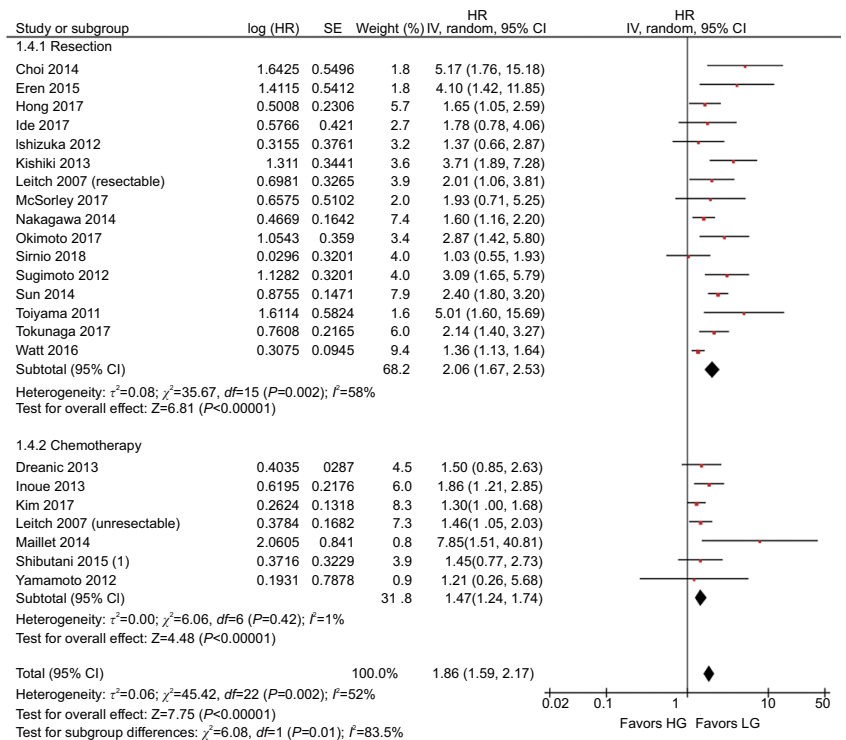

Figure 3 Subgroup analysis showing correlation between GPS and prognosis of CRC patients according to therapeutic strategies.

Note: (A) Overall survival and (B) cancer-specific survival.

Abbreviations: CRC, colorectal cancer; df, degrees of freedom; GPS, Glasgow prognostic score; LG, low group; HG, high group; SE, standard error. 
A

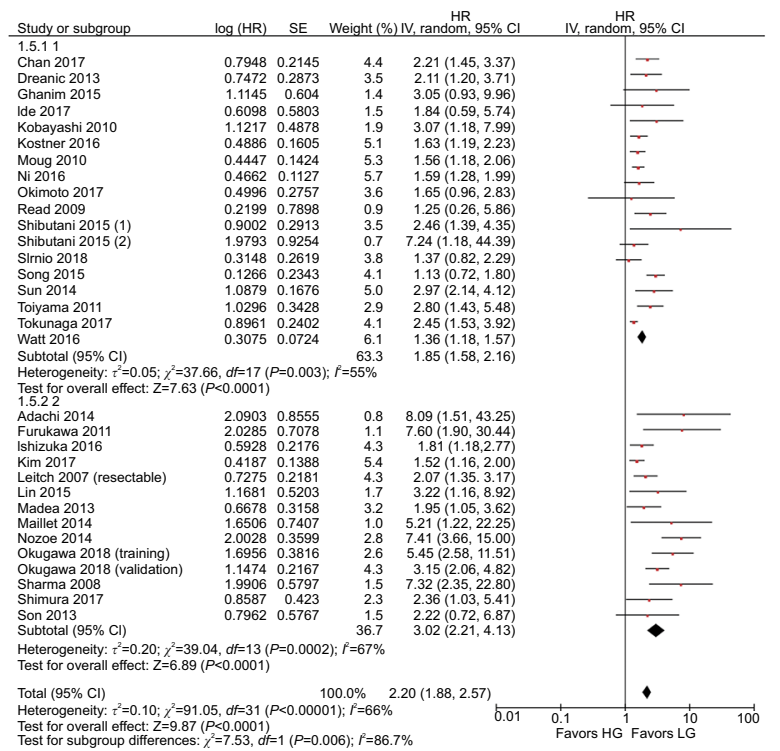

B

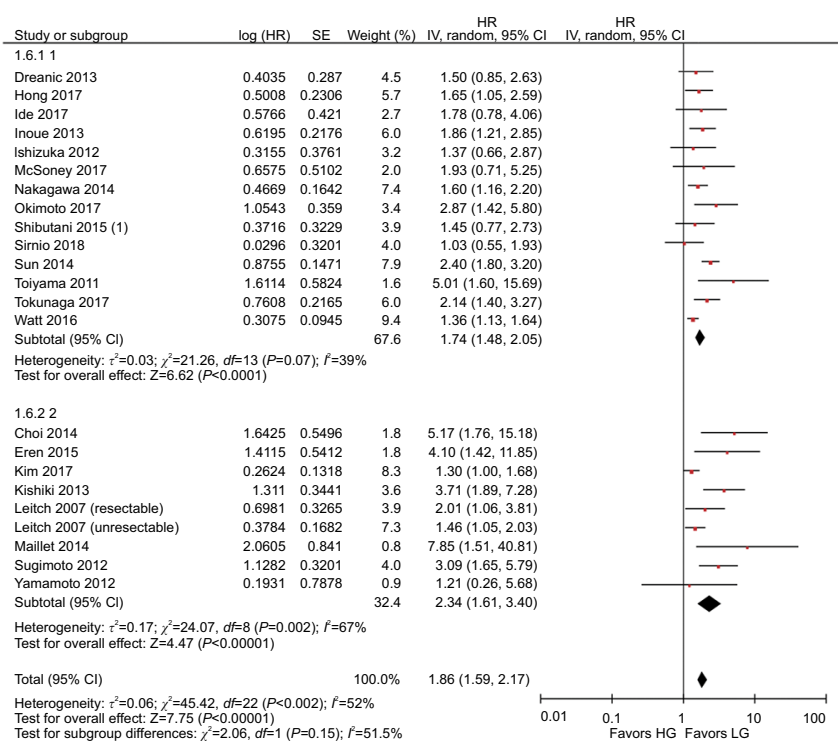

Figure 4 Subgroup analysis showing correlation between GPS and prognosis of CRC patients according to cutoff values.

Note: (A) Overall survival and (B) cancer-specific survival.

Abbreviations: CRC, colorectal cancer; df, degrees of freedom; GPS, Glasgow prognostic score; LG, low group; HG, high group; SE, standard error.

A

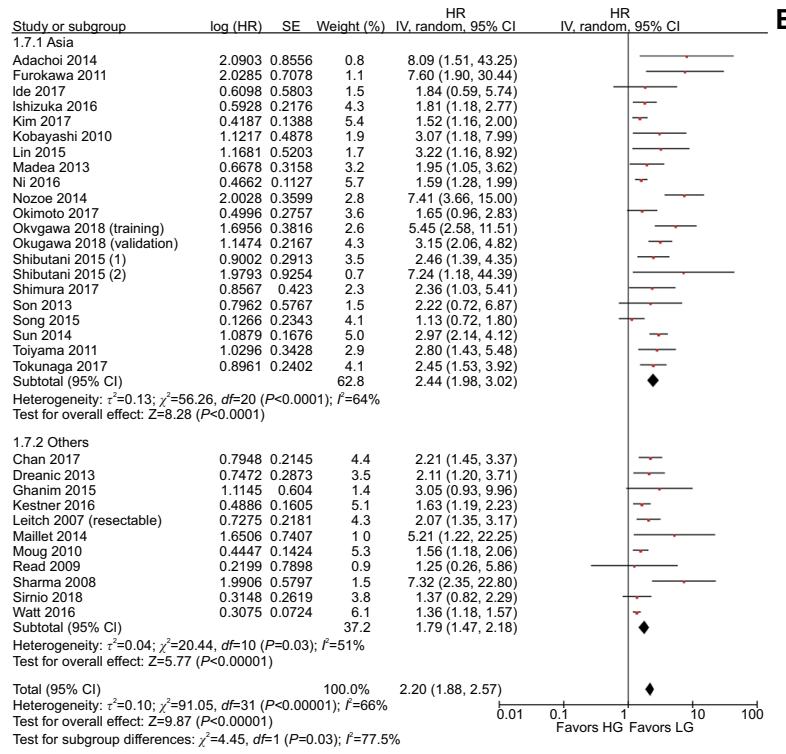

B

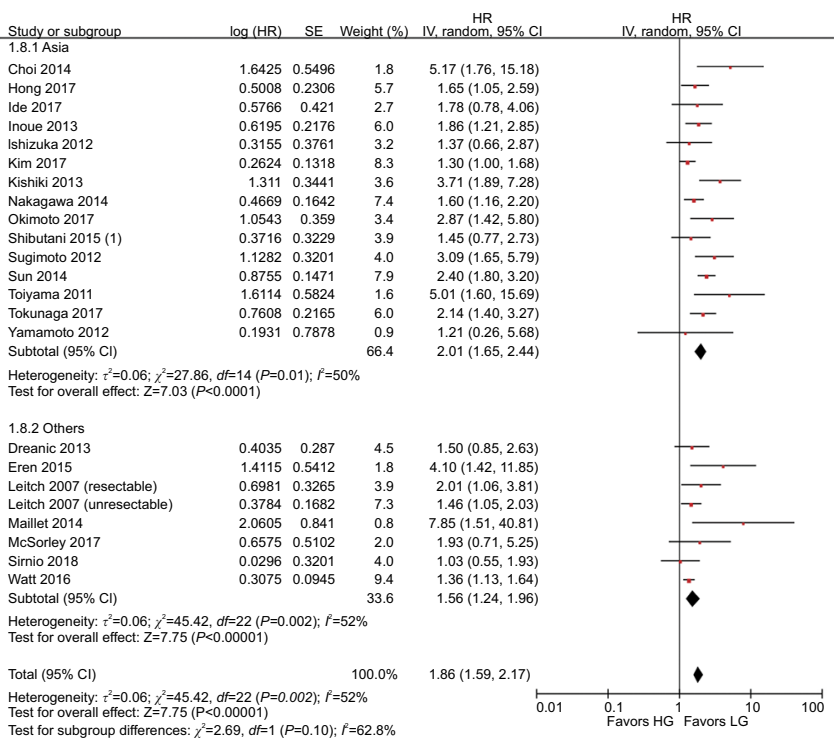

Figure 5 Subgroup analysis showing correlation between GPS and prognosis of CRC patients according to the region of publication.

Note: (A) Overall survival and (B) cancer-specific survival.

Abbreviations: CRC, colorectal cancer; df, degrees of freedom; GPS, Glasgow prognostic score; LG, low group; HG, high group; SE, standard error.

\section{Sensitivity analysis and publication bias}

Sensitivity analysis was conducted by omitting the included studies in sequence to investigate the stability of HR for OS. The results showed that several studies deviated from the center line (Figure 9A). ${ }^{15,51,52,55,58}$ After eliminating these studies, the pooled HRs did not alter significantly (Figure 9B).

The funnel plot showed publication bias in the included studies (Figure 9C). The studies were almost symmetrically distributed around the center line after removing the studies associated with publication bias (Figure 9D). In addition, statistical tests were carried out to evaluate dissymmetry of the funnel plot using Begg ( $\mathrm{z}=1.86, P=0.132)$ and Egger (bias coefficient -0.905 , standard error $0.405, t=1.91$, $P=0.070$ ) tests.

\section{Discussion}

$\mathrm{CRC}$ is one of the most common gastrointestinal malignancies and accounts for $\sim 10 \%$ of all newly diagnosed 
A

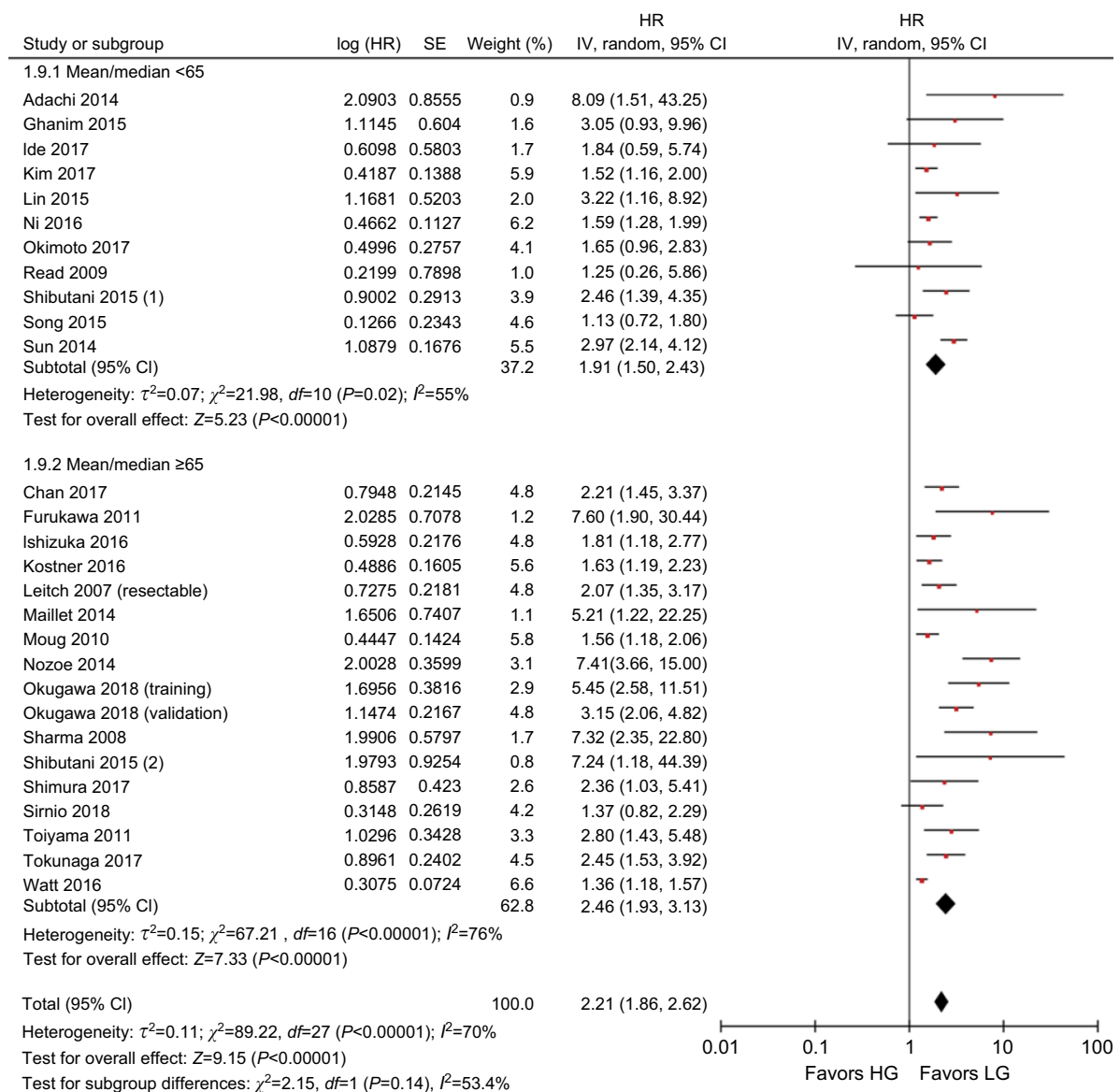

B

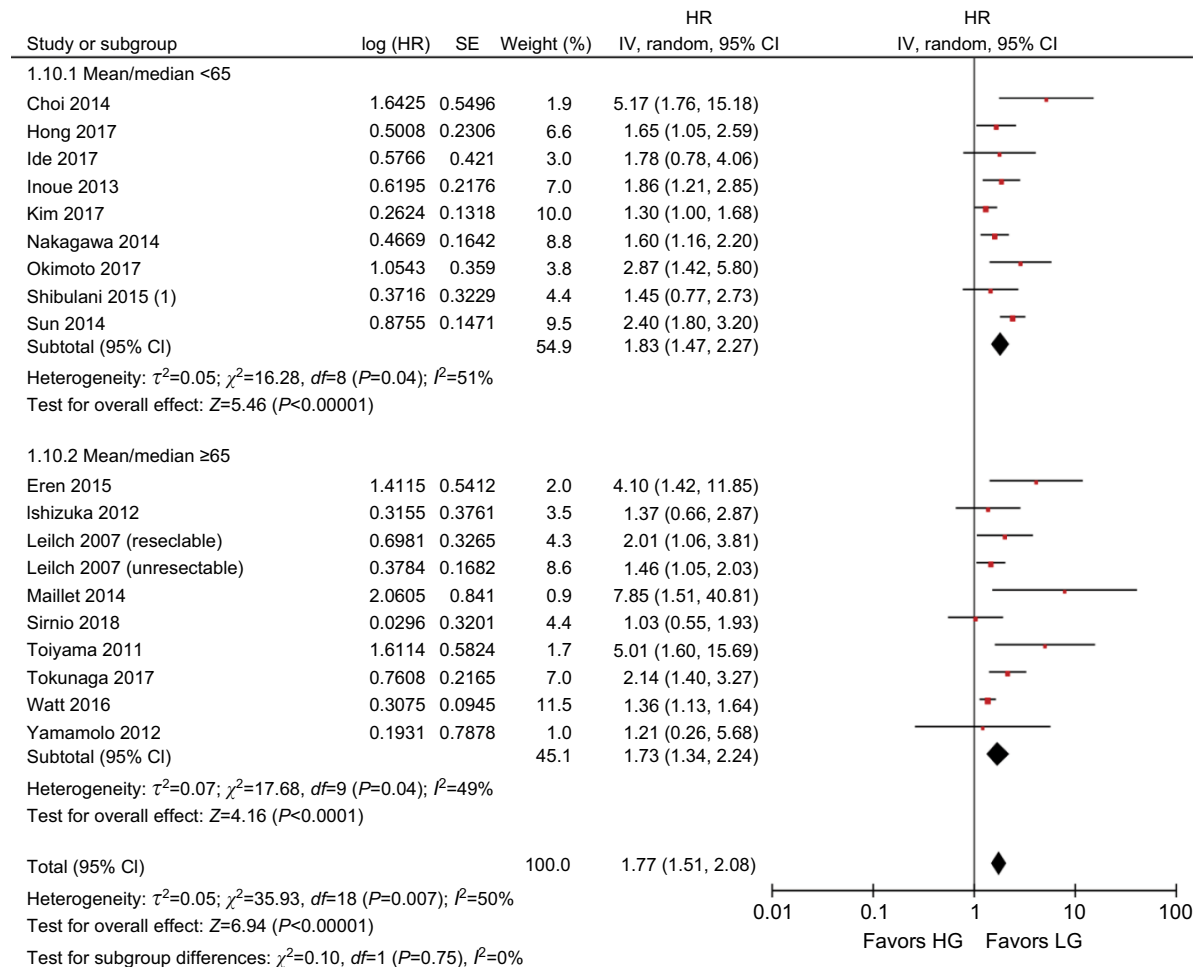

Figure 6 Subgroup analysis showing correlation between GPS and prognosis of CRC patients according to patient age.

Note: (A) Overall survival and (B) cancer-specific survival.

Abbreviations: CRC, colorectal cancer; df, degrees of freedom; GPS, Glasgow prognostic score; LG, low group; HG, high group; SE, standard error. 


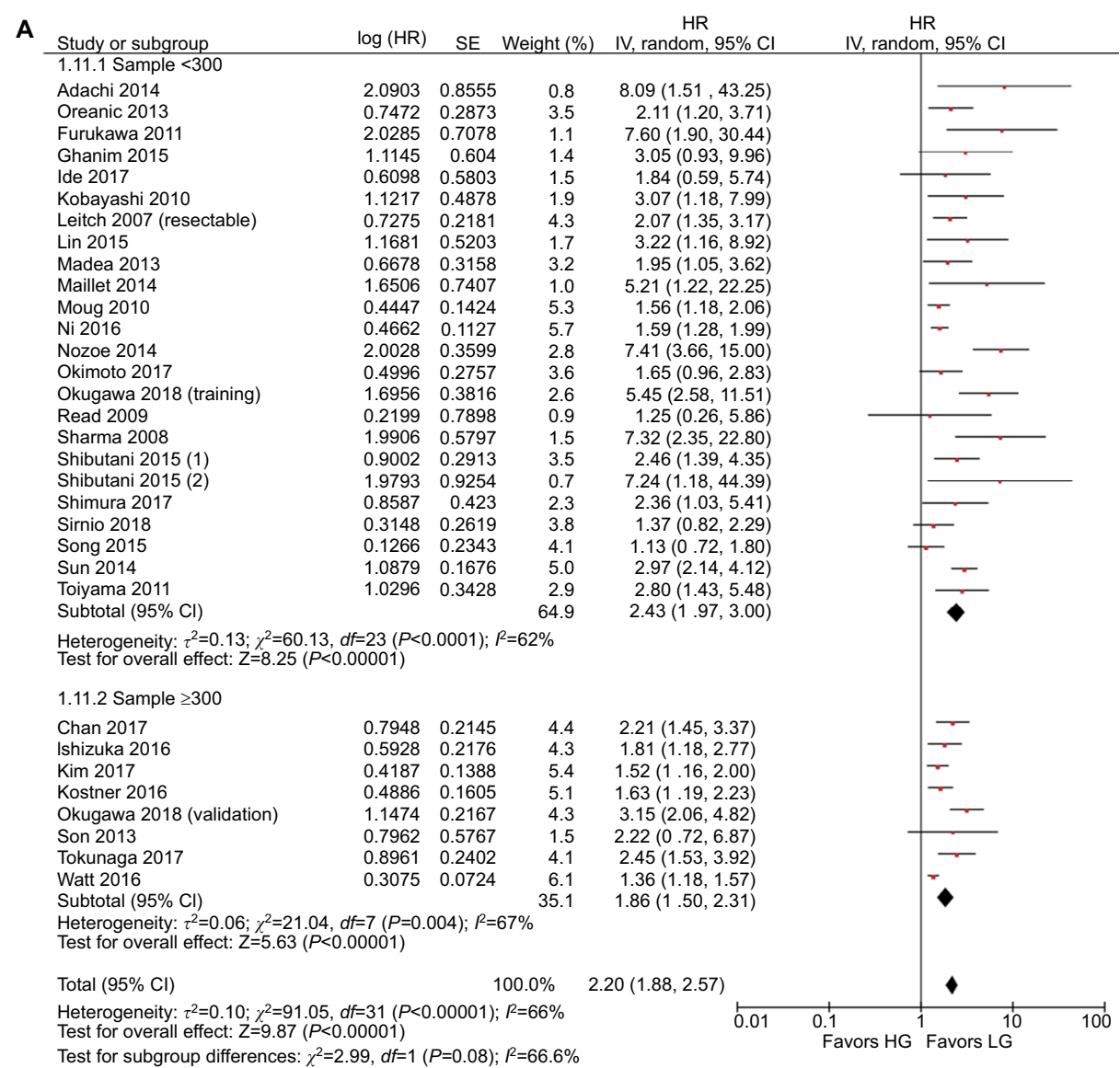

B

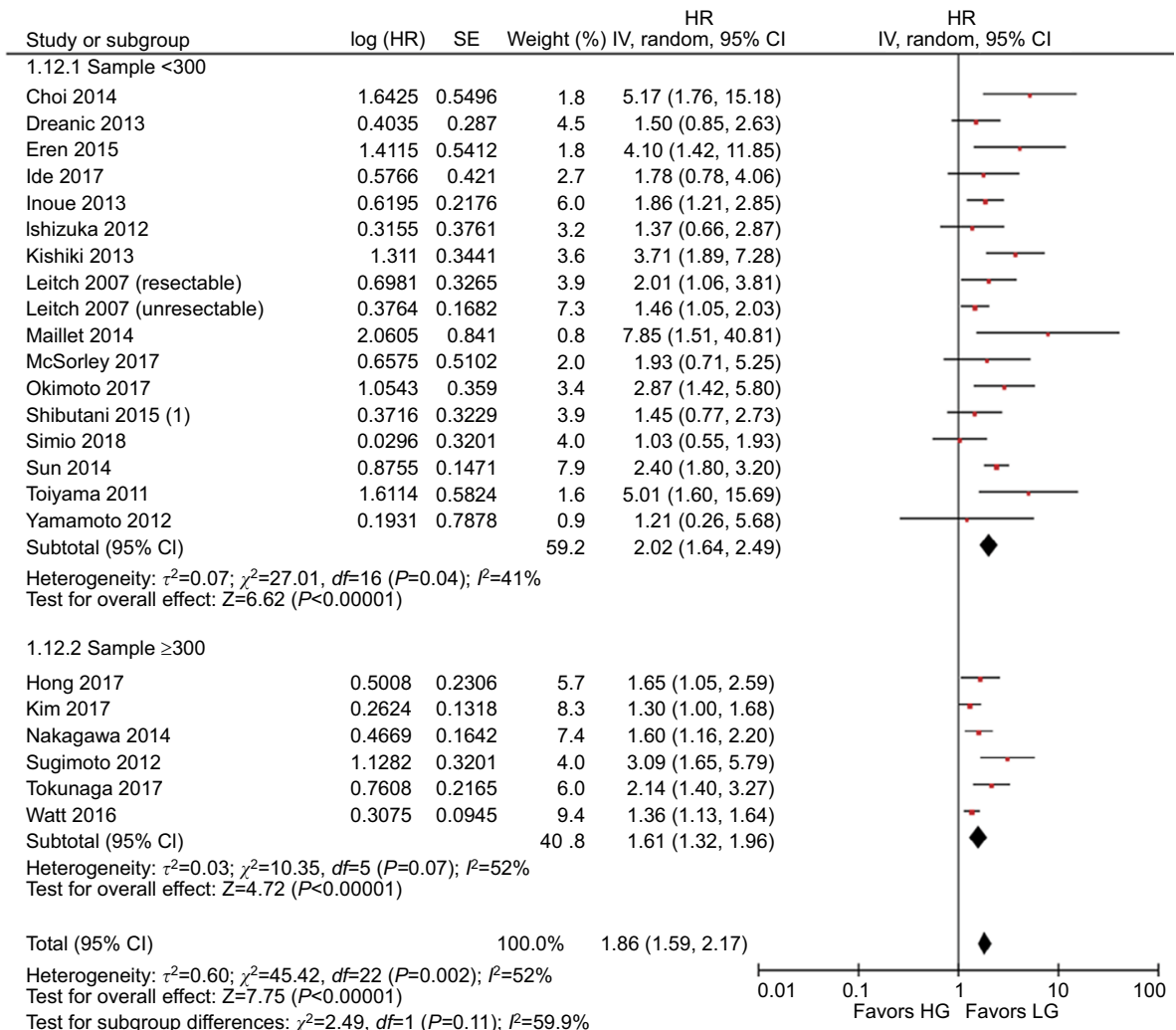

Figure 7 Subgroup analysis showing correlation between GPS and prognosis of CRC patients according to sample size.

Note: (A) Overall survival and (B) cancer-specific survival.

Abbreviations: CRC, colorectal cancer; df, degrees of freedom; GPS, Glasgow prognostic score; LG, low group; HG, high group; SE, standard error. 
A

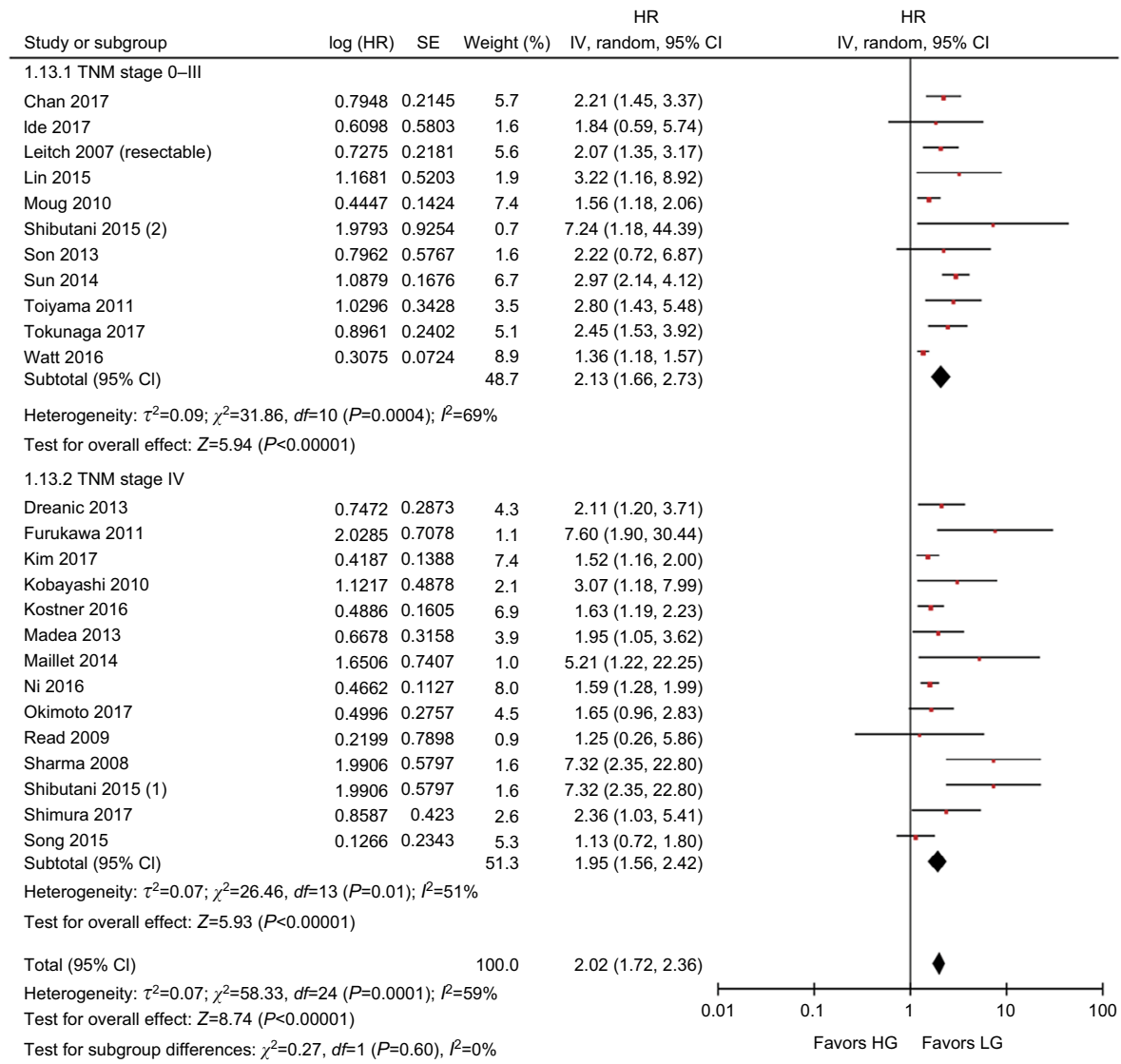

B

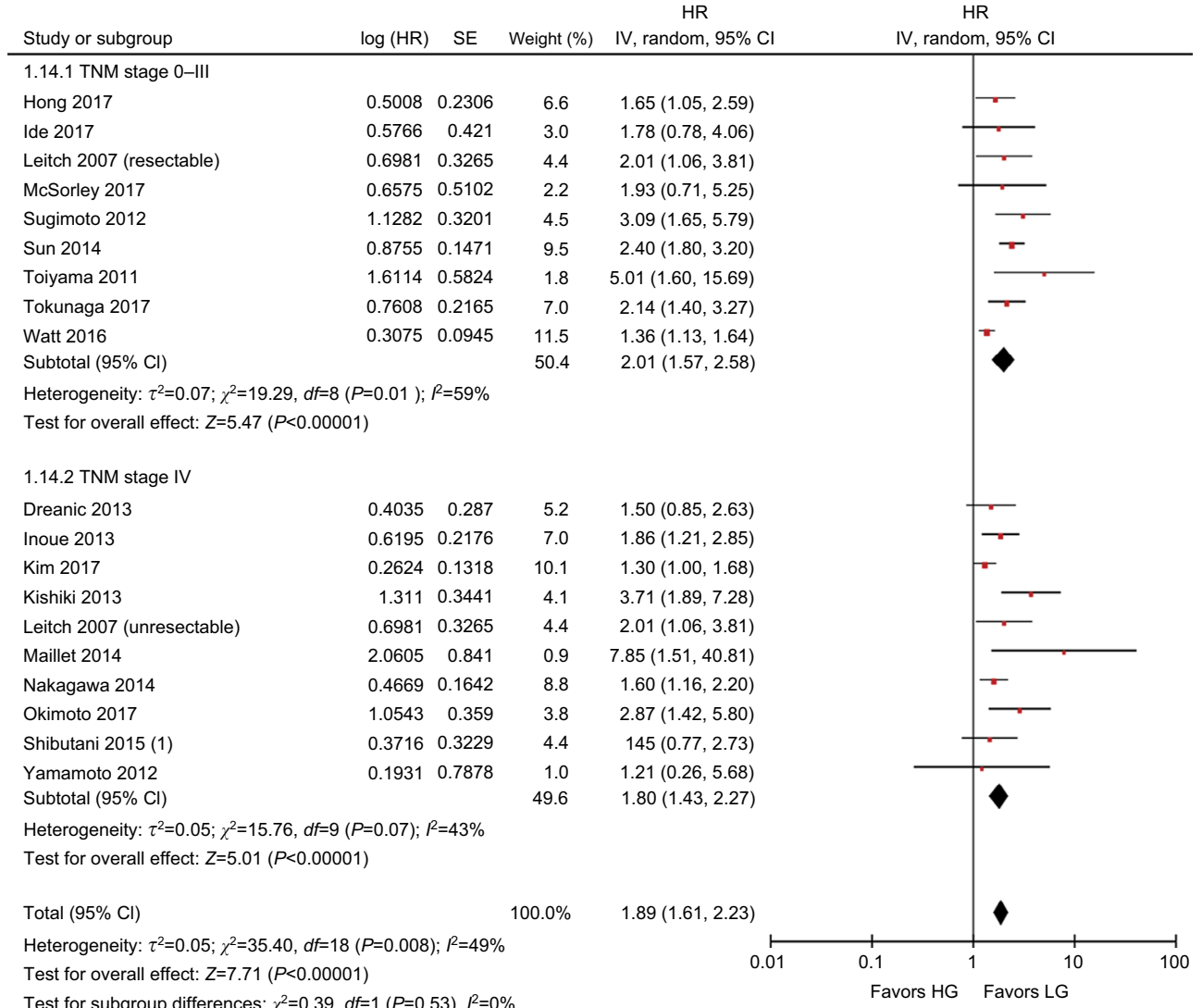

Figure 8 Subgroup analysis showing correlation between GPS and prognosis of CRC patients according to TNM stages.

Note: (A) Overall survival and (B) cancer-specific survival.

Abbreviations: CRC, colorectal cancer; df, degrees of freedom; GPS, Glasgow prognostic score; LG, low group; HG, high group; SE, standard error; TNM, tumor, node, metastases. 
A

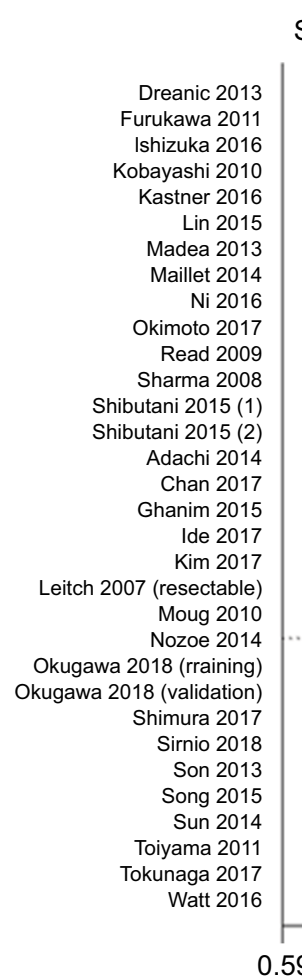

Meta-analysis random effects estimates (linear form)

Study omitted

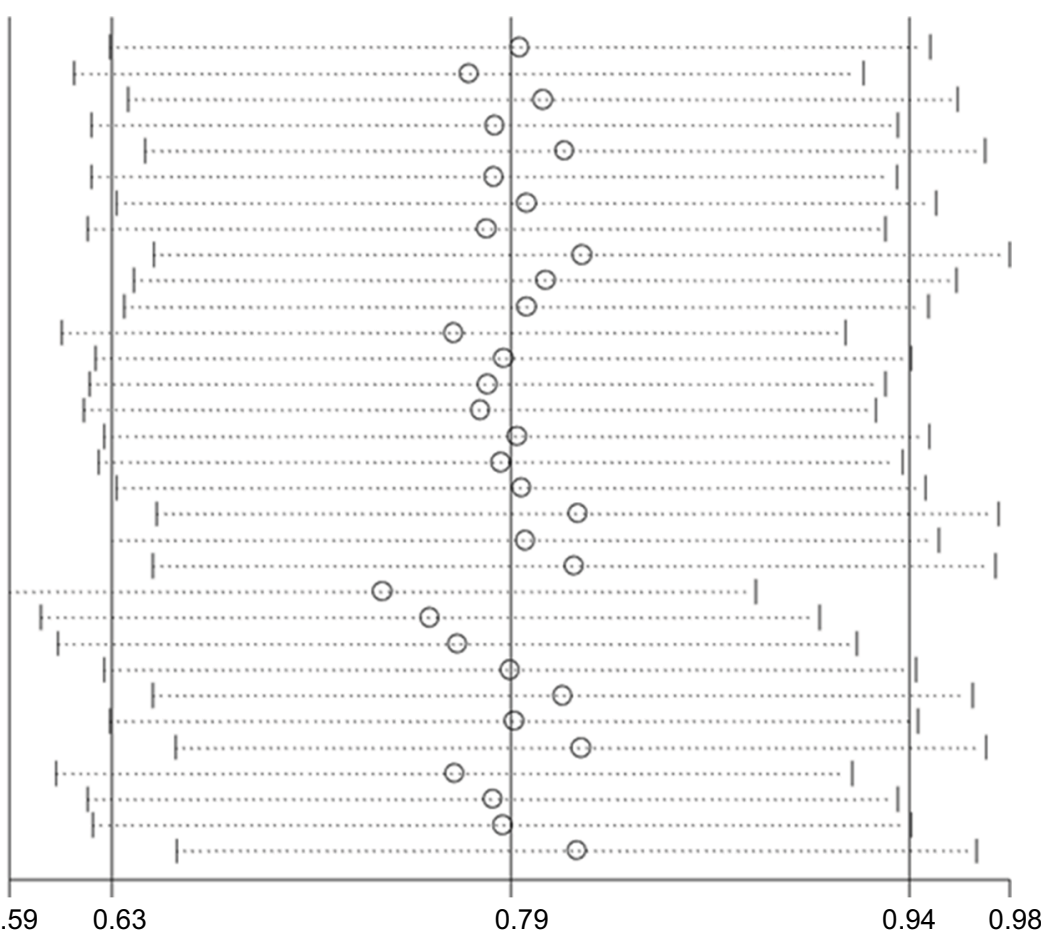

B

Meta-analysis random effects estimates (linear form)

Study omitted

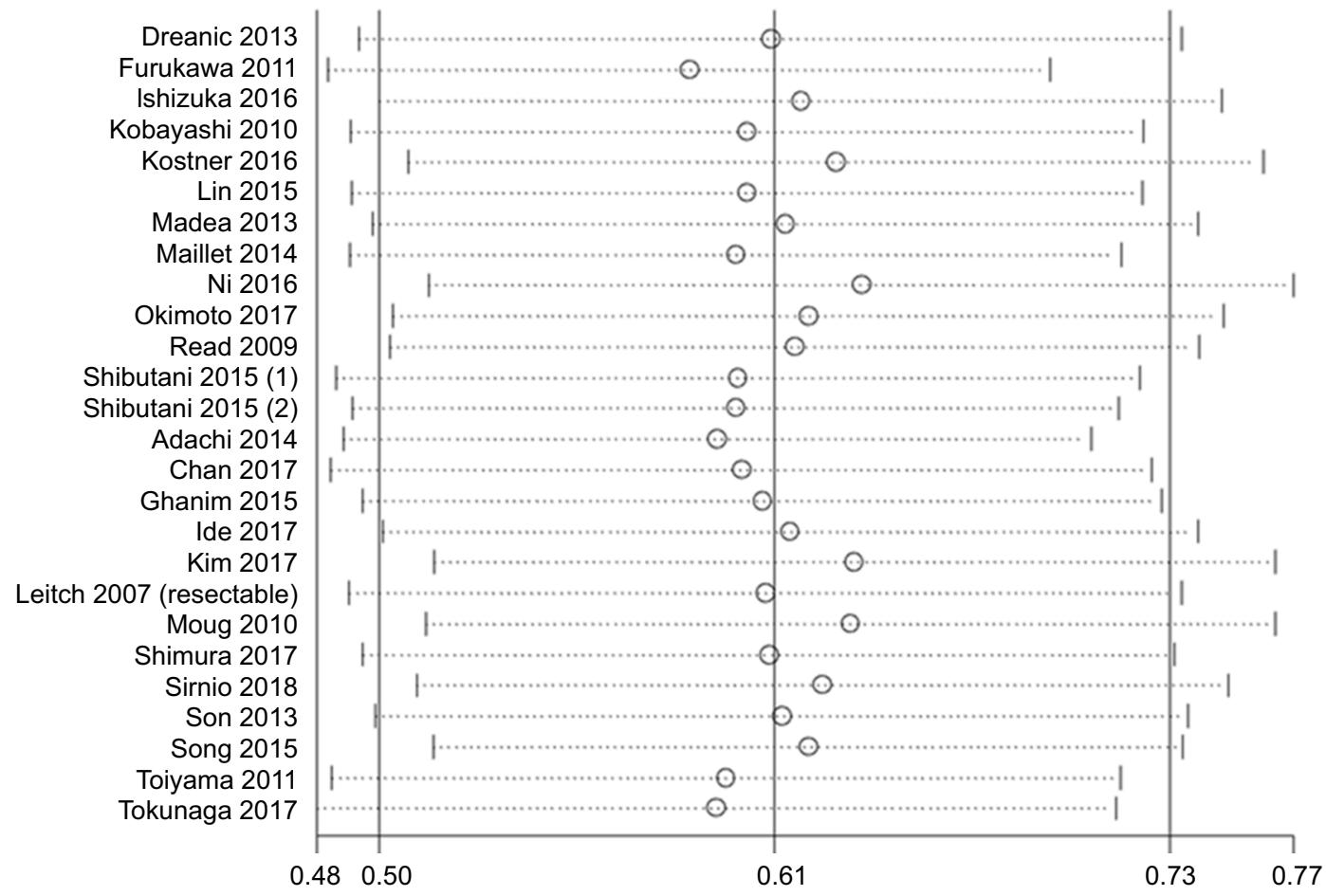

Figure 9 (Continued) 


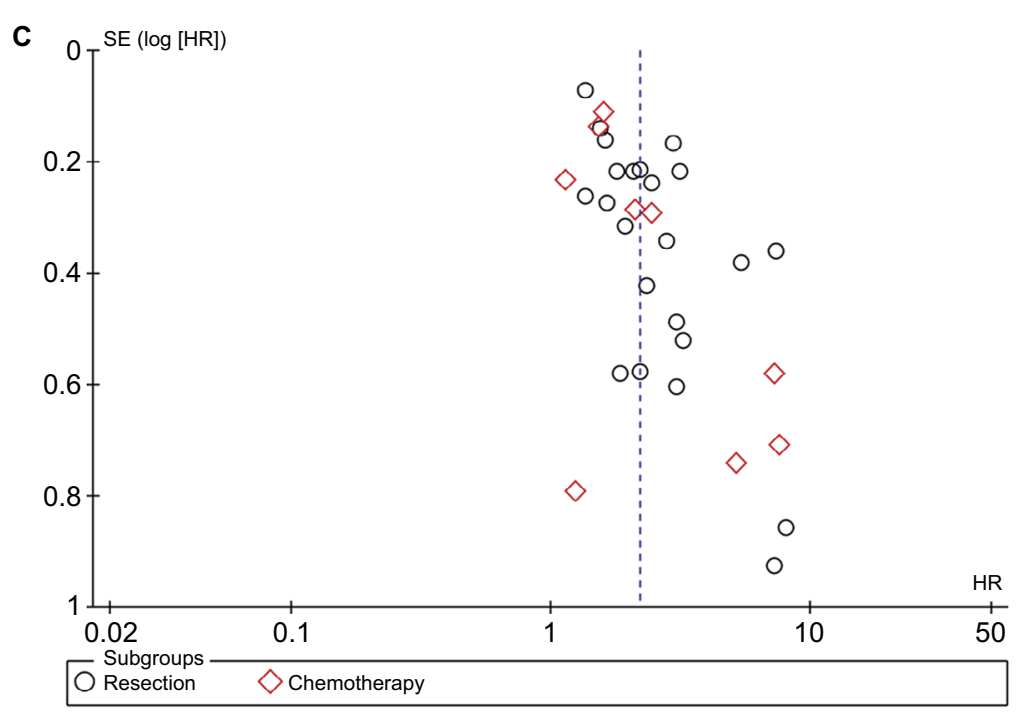

D

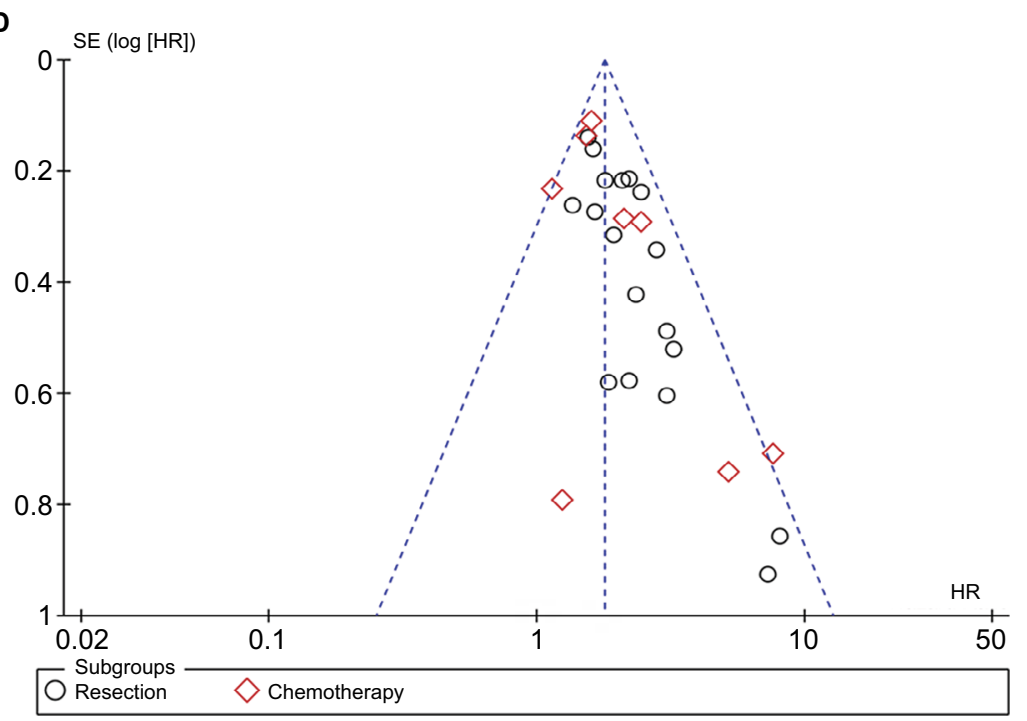

Figure 9 Sensitivity analysis and funnel plot of OS of patients with CRC.

Notes: Sensitivity analysis for $(\mathbf{A})$ overall patients focusing on OS and (B) patients after removing studies with potential publication bias. Funnel plot for (C) overall patients focusing on OS and (D) patients after removing studies with potential publication bias.

Abbreviations: CRC, colorectal cancer; OS, overall survival; SE, standard error.

cancers. The development of therapeutic strategies and improvements in posttreatment quality of life remains a challenge as various factors influence prognosis. An increasing number of prognostic models or indicators have been used to predict the prognosis of patients with CRC. Inflammation-related models including the neutrophil to lymphocyte, platelet to lymphocyte, CRP, and the systemic inflammatory index were developed and demonstrated to be prognostic predictors in recent years. The GPS model was also designed to assess the prognosis of CRC patients. The present study attempted to identify the predictive role of GPS and mGPS by performing a meta-analysis based on the pooled outcomes of research worldwide.

This study included pooled survival outcomes from 43 independent cohorts involving 9,839 CRC patients. The pooled outcomes showed that CRC patients with an increased level of pretreatment GPS or mGPS were associated with worse OS following SR or chemotherapy (HR: 2.20, 95\% CI: $1.88-2.57, P<0.001)$. Moreover, elevated GPS or mGPS levels were also associated with poor CSS (HR: $1.86,95 \% \mathrm{CI}$ : 1.59-2.17, $P<0.001)$. We also conducted subgroup analyses to reduce the heterogeneity. Subgroups were set according to models, therapeutic strategies, cutoff values, geographical regions, age, sample size, and TNM stages. The results of subgroup analyses confirmed the overall outcomes. By conducting the subgroup analyses, heterogeneity was reduced in the subgroups. Thus, the above-mentioned factors should be taken into consideration in future research.

$\mathrm{CRC}$ is one of the most common malignancies, and the prediction of prognosis is an important consideration in the 
establishment of clinical treatment strategies. Recently, the prognostic role of inflammation factors in the management of CRC has been a focus of attention in surgeons. ${ }^{60,61}$ GPS is a prognostic score for cancer patients which calculates prognosis based on the combination of CRP and ALB levels. A growing number of studies have demonstrated the potential predictive value of GPS or mGPS in CRC patients. However, other studies found no correlation between the GPS or mGPS and the survival outcomes of CRC patients. Therefore, it was necessary to perform this systematic review and metaanalysis to draw credible conclusions on the controversial role of GPS in CRC.

As therapeutic strategy was one of the most important factors that influenced the prognostic outcomes of CRC patients, further subgroup analysis was performed to assess the effect of GPS or mGPS in CRC patients according to treatment. The results were consistent with the overall outcomes. Patients with low GPS or mGPS before undergoing SR had a better prognosis and longer CSS. GPS or mGPS was divided into three categories and there was no agreed conclusion on the cutoff values. Thus, subgroup analysis based on cutoff values was performed. The pooled outcomes obtained confirmed the accurate prognostic role of GPS and mGPS using either of the cutoff values. Moreover, patient age was considered another factor that influenced the overall outcomes. Tominaga et al demonstrated that the GPS was not correlated with patient prognosis as all the enrolled patients were over 65 years. ${ }^{62}$ We then conducted another subgroup analysis based on the mean/median patient age of 65 years as most of the patients included in this study were in the 65 years age group. In addition, 65 years was considered to be the dividing line between elderly and middle aged. Subgroup analyses of TNM stages, sample size, and publication regions were also carried out. The results of the subgroup analyses confirmed the overall outcomes and demonstrated the effective prognostic value of GPS or mGPS in predicting survival outcomes in CRC patients.

The results of the sensitivity analysis and funnel plot showed potential publication bias in several studies. After omitting these studies, the OS in the remaining studies was more symmetrically distributed. The reasons for this bias may be due to differences in the baseline characteristics of CRC patients and factors related to the study protocols. Moreover, the differences in detection methods and data storage may have resulted in heterogeneity. Although the random effects model reduced the effect of heterogeneity, the heterogeneity between studies was not abolished.
Two previous meta-analyses reported the prognostic value of GPS or mGPS in CRC patients. ${ }^{63,64}$ Dolan et al examined the evidence for the role of several systemic inflammationbased prognostic scores in patients who underwent SR. ${ }^{63}$ Only 12 studies with 4,739 CRC patients were included in the meta-analysis and no subgroup analyses were conducted. Liu et al performed a meta-analysis by pooling the outcomes of 25 retrospective studies. ${ }^{64}$ The inclusion and exclusion criteria were not rigorous enough and several studies which focused on the prognostic value of GPS were not included. In their study, subgroup analyses were performed based on sample size, cutoff values, and geographical regions. However, they did not include therapeutic strategies, tumor clinical stages, and modifications of the GPS, which accounted for some of the heterogeneity. The present meta-analysis, to our knowledge, is the most comprehensive and included 41 studies. Furthermore, subgroup analyses were also performed.

GPS or mGPS is calculated based on serum CRP and ALB levels. CRP is an acute-phase protein produced in hepatocytes via activation of tumor necrosis factor- $\alpha$ and interleukin- $6 .{ }^{51}$ Several studies have reported that increased serum CRP levels were associated with poor outcomes in a variety of solid tumors. ${ }^{65-67}$ In contrast, hypoalbuminemia is considered to be an indicator of malnutrition and cachexia. Two studies have shown that hypoalbuminemia was associated with poor outcomes in various cancers. ${ }^{68,69}$ The GPS or mGPS enables better appreciation of systemic inflammation or malnutrition in CRC patients. This study pooled outcomes and then drew reliable conclusions with regard to the prognostic value of the GPS or mGPS. Based on these conclusions, the GPS or mGPS should be highlighted in the clinical management of CRC. For patients with elevated GPS or mGPS, a management protocol for systemic inflammatory response via the tumor-host interaction during the postoperative course is urgently needed to improve their prognosis. ${ }^{51}$

The present meta-analysis was performed based on the largest patient sample available to date. However, the study had several limitations. Firstly, most of the included studies were retrospectively designed. This increased the risk of bias due to inadequate random blinding and sequencing. Secondly, even though subgroup analyses were performed, heterogeneity still existed among subgroups. This may have been due to a variety of baseline characteristics and follow-up information. Furthermore, the overall outcomes might be overvalued due to unpublished studies which had negative data. Finally, all included studies were written in English, and this may have resulted in publication bias. These limitations should be taken into consideration in further studies to confirm our results. 
Nevertheless, the present meta-analysis was conducted at an appropriate time based on sufficient studies with enough data to investigate the prognostic value of GPS or mGPS in CRC patients. A meta-analysis is a statistical inspection of scientific studies, and its level of evidence is considered to be superior to that of individual studies. ${ }^{70}$ The results of this meta-analysis are encouraging although multiple strategies were used to identify relevant studies, with strict criteria used for study inclusion and evaluation. Subgroup analyses were performed to minimize heterogeneity due to different treatment modalities, cutoff values, regions, ages, and tumor stages. In addition, our study is the most comprehensive and up-to-date systematic review and meta-analysis which focused on the role of GPS or mGPS in predicting the prognosis of CRC.

\section{Conclusion}

The present study indicated that pretreatment GPS or mGPS was an accurate prognostic predictor in patients with CRC. Patients with elevated pretreatment GPS or mGPS were associated with worse prognosis. Subgroup analyses confirmed the overall outcomes. Pretreatment GPS or mGPS should be identified as an important parameter in the management of CRC.

\section{Disclosure}

The authors report no conflicts of interest in this work.

\section{References}

1. Haggar FA, Boushey RP. Colorectal cancer epidemiology: incidence, mortality, survival, and risk factors. Clin Colon Rectal Surg. 2009;22(4):191-197.

2. Arnold M, Sierra MS, Laversanne M, Soerjomataram I, Jemal A, Bray F. Global patterns and trends in colorectal cancer incidence and mortality. Gut. 2017;66(4):683-691.

3. Global Burden of Disease Cancer Collaboration, Fitzmaurice C, Dicker D, et al. The global burden of cancer 2013. JAMA Oncol. 2015;1(4):505-527.

4. Desantis CE, Lin CC, Mariotto AB, et al. Cancer treatment and survivorship statistics, 2014. CA Cancer J Clin. 2014;64(4):252-271.

5. Glynne-Jones R, Wyrwicz L, Tiret E, et al. Rectal cancer: ESMO Clinical Practice Guidelines for diagnosis, treatment and follow-up. Ann Oncol. 2017;28(Suppl_4):iv22-iv40.

6. Ueno H, Mochizuki H, Akagi Y, et al. Optimal colorectal cancer staging criteria in TNM classification. J Clin Oncol. 2012;30(13):1519-1526.

7. Wittekind C, Oberschmid B. TNM classification of malignant tumors 2010: General aspects and amendments in the general section. Pathologe. 2010;31(5):333336-333338.

8. Roxburgh CS, Salmond JM, Horgan PG, Oien KA, McMillan DC. Comparison of the prognostic value of inflammation-based pathologic and biochemical criteria in patients undergoing potentially curative resection for colorectal cancer. Ann Surg. 2009;249(5):788-793.

9. Roxburgh CS, McMillan DC. Cancer and systemic inflammation: treat the tumour and treat the host. Br J Cancer. 2014;110(6): 1409-1412.
10. Aliustaoglu M, Bilici A, Ustaalioglu BB, et al. The effect of peripheral blood values on prognosis of patients with locally advanced gastric cancer before treatment. Med Oncol. 2010;27(4):1060-1065.

11. Sagawa M, Yoshimatsu K, Yokomizo H, et al. Worse preoperative status based on inflammation and host immunity is a risk factor for surgical site infections in colorectal cancer surgery. J Nippon Med Sch. 2017;84(5):224-230.

12. Kinoshita A, Onoda $H$, Imai N, et al. Comparison of the prognostic value of inflammation-based prognostic scores in patients with hepatocellular carcinoma. Br J Cancer. 2012;107(6):988-993.

13. Ishizuka M, Nagata H, Takagi K, Iwasaki Y, Kubota K. Inflammationbased prognostic system predicts postoperative survival of colorectal cancer patients with a normal preoperative serum level of carcinoembryonic antigen. Ann Surg Oncol. 2012;19(11):3422-3431.

14. Leitch EF, Chakrabarti M, Crozier JE, et al. Comparison of the prognostic value of selected markers of the systemic inflammatory response in patients with colorectal cancer. Br J Cancer. 2007;97(9): $1266-1270$.

15. Nozoe T, Matono R, Ijichi H, Ohga T, Ezaki T. Glasgow Prognostic Score (GPS) can be a useful indicator to determine prognosis of patients with colorectal carcinoma. Int Surg. 2014;99(5):512-517.

16. Sugimoto K, Komiyama H, Kojima Y, Goto M, Tomiki Y, Sakamoto $\mathrm{K}$. Glasgow prognostic score as a prognostic factor in patients undergoing curative surgery for colorectal cancer. Dig Surg. 2012;29(6): 503-509.

17. Choi KW, Hong SW, Chang YG, et al. Inflammation-based score (Glasgow prognostic score) as an independent prognostic factor in colorectal cancer patients. Ann Surg Treat Res. 2014;86(6):309-313.

18. Forrest LM, McMillan DC, McArdle CS, Angerson WJ, Dunlop DJ. Evaluation of cumulative prognostic scores based on the systemic inflammatory response in patients with inoperable non-small-cell lung cancer. Br J Cancer. 2003;89(6):1028-1030.

19. Read JA, Choy ST, Beale PJ, Clarke SJ. Evaluation of nutritional and inflammatory status of advanced colorectal cancer patients and its correlation with survival. Nutr Cancer. 2006;55(1):78-85.

20. Adachi T, Hinoi T, Hattori M, et al. The modified Glasgow prognostic score for early mortality in patients with synchronous peritoneal carcinomatosis from colorectal cancer. Surg Today. 2015;45(11):1396-1403.

21. Kishiki T, Masaki T, Matsuoka H, et al. Modified Glasgow prognostic score in patients with incurable stage IV colorectal cancer. Am J Surg. 2013;206(2):234-240.

22. Kurahara H, Maemura K, Mataki Y, et al. Prognostication by inflammation-based score in patients with locally advanced pancreatic cancer treated with chemoradiotherapy. Pancreatology. 2015;15(6):688-693.

23. Crumley AB, McMillan DC, McKernan M, McDonald AC, Stuart RC. Evaluation of an inflammation-based prognostic score in patients with inoperable gastro-oesophageal cancer. Br J Cancer. 2006;94(5): 637-641.

24. Ishizuka M, Nagata H, Takagi K, Iwasaki Y, Shibuya N, Kubota $\mathrm{K}$. Clinical significance of the C-reactive protein to albumin ratio for survival after surgery for colorectal cancer. Ann Surg Oncol. 2016;23(3):900-907.

25. Okimoto S, Kobayashi T, Tashiro H, et al. Significance of the Glasgow Prognostic Score for patients with colorectal liver metastasis. Int J Surg. 2017;42:209-214

26. Sirniö P, Tuomisto A, Tervahartiala T, et al. High-serum MMP-8 levels are associated with decreased survival and systemic inflammation in colorectal cancer. Br J Cancer. 2018;119(2):213-219.

27. Lan X, Zhang MM, Pu CL, et al. Impact of human leukocyte antigen mismatching on outcomes of liver transplantation: a meta-analysis. World J Gastroenterol. 2010;16(27):3457-3464.

28. Li H, Zhang JB, Chen XL, et al. Different techniques for harvesting grafts for living donor liver transplantation: A systematic review and meta-analysis. World J Gastroenterol. 2017;23(20):3730-3743.

29. Li H, Zheng J, Cai JY, et al. Laparoscopic $V S$ open hepatectomy for hepatolithiasis: An updated systematic review and meta-analysis. World J Gastroenterol. 2017;23(43):7791-7806. 
30. Hayden JA, van der Windt DA, Cartwright JL, Côté P, Bombardier C. Assessing bias in studies of prognostic factors. Ann Intern Med. 2013;158(4):280-286.

31. Shibutani M, Maeda K, Nagahara H, et al. Significance of markers of systemic inflammation for predicting survival and chemotherapeutic outcomes and monitoring tumor progression in patients with unresectable metastatic colorectal cancer. Anticancer Res. 2015;35(9):5037-5046.

32. Shimura T, Toiyama Y, Saigusa S, et al. Inflammation-based prognostic scores as indicators to select candidates for primary site resection followed by multimodal therapy among colorectal cancer patients with multiple metastases. Int J Clin Oncol. 2017;22(4):758-766.

33. Chan JC, Chan DL, Diakos CI, et al. The lymphocyte-to-monocyte ratio is a superior predictor of overall survival in comparison to established biomarkers of resectable colorectal cancer. Ann Surg. 2017;265(3):539-546.

34. Dréanic J, Maillet M, Dhooge M, et al. Prognostic value of the Glasgow Prognostic Score in metastatic colorectal cancer in the era of anti-EGFR therapies. Med Oncol. 2013;30(3):656.

35. Eren T, Burcu B, Tombalak E, et al. Clinical significance of the glasgow prognostic score for survival after colorectal cancer surgery. $J$ Gastrointest Surg. 2016;20(6):1231-1238.

36. Furukawa K, Shiba H, Haruki K, et al. The Glasgow prognostic score is valuable for colorectal cancer with both synchronous and metachronous unresectable liver metastases. Oncol Lett. 2012;4(2):324-328.

37. Ghanim B, Schweiger T, Jedamzik J, et al. Elevated inflammatory parameters and inflammation scores are associated with poor prognosis in patients undergoing pulmonary metastasectomy for colorectal cancer. Interact Cardiovasc Thorac Surg. 2015;21(5):616-623.

38. Hong T, Shen D, Chen X, Cai D, Wu X, Hua D. A novel systematic inflammation related index is prognostic in curatively resected nonmetastatic colorectal cancer. Am J Surg. 2018;216(3):450-457.

39. Ide S, Toiyama $\mathrm{Y}$, Okugawa $\mathrm{Y}$, et al. Clinical significance of $\mathrm{C}$-reactive protein-to-albumin ratio with rectal cancer patient undergoing chemoradiotherapy followed by surgery. Anticancer Res. 2017;37(10):5797-5804.

40. Inoue $Y$, Iwata $T$, Okugawa $Y$, et al. Prognostic significance of a systemic inflammatory response in patients undergoing multimodality therapy for advanced colorectal cancer. Oncology. 2013;84(2):100-107.

41. Kim IH, Lee JE, Yang JH, Jeong JW, Ro S, Lee MA. Clinical significance of changes in systemic inflammatory markers and carcinoembryonic antigen levels in predicting metastatic colorectal cancer prognosis and chemotherapy response. Asia Pac J Clin Oncol. 2018;14(3):239-246.

42. Kobayashi T, Teruya M, Kishiki T, et al. Elevated C-reactive protein and hypoalbuminemia measured before resection of colorectal liver metastases predict postoperative survival. Dig Surg. 2010;27(4):285-290.

43. Køstner AH, Kersten C, Löwenmark T, et al. The prognostic role of systemic inflammation in patients undergoing resection of colorectal liver metastases: C-reactive protein (CRP) is a strong negative prognostic biomarker. J Surg Oncol. 2016;114(7):895-899.

44. Lin MS, Huang JX, Yu H. Prognostic significance of Glasgow prognostic score in patients with stage II colorectal cancer. Int J Clin Exp Med. 2015;8(10):19138-19143.

45. Maeda K, Shibutani M, Otani H, et al. Prognostic value of preoperative inflammation-based prognostic scores in patients with stage IV colorectal cancer who undergo palliative resection of asymptomatic primary tumors. Anticancer Res. 2013;33(12):5567-5573.

46. Maillet M, Dréanic J, Dhooge M, et al. The predictive and prognostic value of the Glasgow Prognostic Score in metastatic colorectal carcinoma patients receiving bevacizumab. Anticancer Drugs. 2014;25(10):1215-1219.

47. McSorley ST, Khor BY, Tsang K, et al. The relationship between ${ }^{18} \mathrm{~F}$-FDG-PETCT-derived markers of tumour metabolism and systemic inflammation in patients with recurrent disease following surgery for colorectal cancer. Colorectal Dis. 2018;20(5):407-415.

48. Moug SJ, McColl G, Lloyd SM, Wilson G, Saldanha JD, Diament $\mathrm{RH}$. Comparison of positive lymph node ratio with an inflammationbased prognostic score in colorectal cancer. Br J Surg. 2011;98(2): 282-286.
49. Nakagawa K, Tanaka K, Nojiri K, et al. The modified Glasgow prognostic score as a predictor of survival after hepatectomy for colorectal liver metastases. Ann Surg Oncol. 2014;21(5):1711-1718.

50. Ni X, Wu P, Wu J, et al. C-reactive protein/albumin ratio as a predictor of survival of metastatic colorectal cancer patients receiving chemotherapy. Int J Clin Exp Pathol. 2016;9:5525-5534.

51. Okugawa Y, Shirai Y, Toiyama Y, et al. Clinical Burden of Modified Glasgow Prognostic Scale in Colorectal Cancer. Anticancer Res. 2018;38(3):1599-1610.

52. Sharma R, Zucknick M, London R, Kacevska M, Liddle C, Clarke SJ. Systemic inflammatory response predicts prognosis in patients with advanced-stage colorectal cancer. Clin Colorectal Cancer. 2008;7(5):331-337.

53. Son HJ, Park JW, Chang HJ, et al. Preoperative plasma hyperfibrinogenemia is predictive of poor prognosis in patients with nonmetastatic colon cancer. Ann Surg Oncol. 2013;20(9):2908-2913.

54. Song A, Eo W, Lee S. Comparison of selected inflammation-based prognostic markers in relapsed or refractory metastatic colorectal cancer patients. World J Gastroenterol. 2015;21(43):12410-12420.

55. Sun ZQ, Han XN, Wang HJ, et al. Prognostic significance of preoperative fibrinogen in patients with colon cancer. World J Gastroenterol. 2014;20(26):8583-8591.

56. Toiyama Y, Miki C, Inoue Y, Tanaka K, Mohri Y, Kusunoki M. Evaluation of an inflammation-based prognostic score for the identification of patients requiring postoperative adjuvant chemotherapy for stage II colorectal cancer. Exp Ther Med. 2011;2(1):95-101.

57. Tokunaga R, Sakamoto Y, Nakagawa S, et al. Comparison of systemic inflammatory and nutritional scores in colorectal cancer patients who underwent potentially curative resection. Int J Clin Oncol. 2017;22(4):740-748.

58. Watt DG, McSorley ST, Park JH, Horgan PG, McMillan DC. A postoperative systemic inflammation score predicts short- and long-term outcomes in patients undergoing surgery for colorectal cancer. Ann Surg Oncol. 2017;24(4):1100-1109.

59. Yamamoto M, Yoshinaga K, Matsuyama A, et al. Plasma D-dimer level as a mortality predictor in patients with advanced or recurrent colorectal cancer. Oncology. 2012;83(1):10-15.

60. Lumachi F, Basso SM, Santeufemia DA, Ermani M, Lo Re G, Chiara GB. Preoperative serum C-reactive protein and its prognostic significance in patients with stage III-IV colorectal cancer. Anticancer Res. 2014;34(12):7263-7266.

61. Ying HQ, Deng QW, He BS, et al. The prognostic value of preoperative NLR, d-NLR, PLR and LMR for predicting clinical outcome in surgical colorectal cancer patients. Med Oncol. 2014;31(12):305.

62. Tominaga $\mathrm{T}$, Nonaka $\mathrm{T}$, Takeshita $\mathrm{H}$, et al. The charlson comorbidity index as an independent prognostic factor in older colorectal cancer patients. Indian J Surg. 2018;80(1):54-60.

63. Dolan RD, Lim J, McSorley ST, Horgan PG, McMillan DC. The role of the systemic inflammatory response in predicting outcomes in patients with operable cancer: Systematic review and meta-analysis. Sci Rep. 2017;7(1): 16717.

64. Liu Y, He X, Pan J, Chen S, Wang L. Prognostic role of Glasgow prognostic score in patients with colorectal cancer: evidence from population studies. Sci Rep. 2017;7(1):6144.

65. Jagdev SP, Gregory W, Vasudev NS, et al. Improving the accuracy of pre-operative survival prediction in renal cell carcinoma with C-reactive protein. Br J Cancer. 2010;103(11):1649-1656.

66. Yamamoto M, Saito H, Uejima C, et al. Prognostic value of the combination of pre- and postoperative C-reactive protein in colorectal cancer patients. Surg Today. 2018;48(11):986-993.

67. Oh TK, Choi YR, Cho JY, et al. The high-sensitivity C-reactive protein/ albumin ratio predicts long-term oncologic outcomes after curative resection for hepatocellular carcinoma. J Clin Med. 2018;7(6):139.

68. McMillan DC, Crozier JE, Canna K, Angerson WJ, McArdle CS. Evaluation of an inflammation-based prognostic score (GPS) in patients undergoing resection for colon and rectal cancer. Int $J$ Colorectal Dis. 2007;22(8):881-886. 
69. Li X, Qin S, Sun X, et al. Prognostic significance of albumin-globulin score in patients with operable non-small-cell lung cancer. Ann Surg Oncol. 2018;25(12):3647-3659.
70. Dhir M, Lyden ER, Wang A, Smith LM, Ullrich F, Are C. Influence of margins on overall survival after hepatic resection for colorectal metastasis: a meta-analysis. Ann Surg. 2011;254(2):234-242. 


\section{Supplementary material}

Table SI Summary of risk of bias

\begin{tabular}{|c|c|c|c|c|c|c|}
\hline Study & $\begin{array}{l}\text { Study } \\
\text { participation }\end{array}$ & $\begin{array}{l}\text { Study } \\
\text { attrition }\end{array}$ & $\begin{array}{l}\text { Prognostic } \\
\text { factor } \\
\text { measurement }\end{array}$ & $\begin{array}{l}\text { Outcome } \\
\text { measurement }\end{array}$ & $\begin{array}{l}\text { Study } \\
\text { confounding }\end{array}$ & $\begin{array}{l}\text { Statistical } \\
\text { analysis and } \\
\text { reporting }\end{array}$ \\
\hline Adachi et al ${ }^{\prime}$ & - & - & \pm & - & - & \pm \\
\hline Chan et al ${ }^{2}$ & - & - & - & - & \pm & \pm \\
\hline Choi et al ${ }^{3}$ & - & - & \pm & - & \pm & - \\
\hline Dréanic et $\mathrm{al}^{4}$ & - & \pm & - & \pm & \pm & - \\
\hline Eren et $\mathrm{al}^{5}$ & - & - & \pm & - & \pm & - \\
\hline Furukawa et $\mathrm{al}^{6}$ & \pm & \pm & - & - & - & - \\
\hline Ghanim et al ${ }^{7}$ & \pm & \pm & - & - & - & - \\
\hline Hong et $\mathrm{al}^{8}$ & - & - & - & - & \pm & - \\
\hline Ide et al ${ }^{9}$ & - & - & \pm & - & \pm & - \\
\hline Inoue et al ${ }^{10}$ & - & - & - & - & \pm & - \\
\hline Ishizuka et al" & - & - & - & - & - & - \\
\hline Ishizuka et al ${ }^{12}$ & - & - & - & - & \pm & \pm \\
\hline Kim et al ${ }^{13}$ & - & - & - & - & \pm & - \\
\hline Kishiki et al ${ }^{14}$ & - & - & - & - & \pm & - \\
\hline Kobayashi et al ${ }^{15}$ & \pm & - & - & - & \pm & - \\
\hline Køstner et al ${ }^{16}$ & - & - & - & - & \pm & - \\
\hline Leitch et al ${ }^{17}$ & - & - & - & - & - & \pm \\
\hline Lin et al ${ }^{18}$ & \pm & - & - & - & \pm & \pm \\
\hline Maeda et al ${ }^{19}$ & \pm & - & - & - & \pm & - \\
\hline Maillet et $\mathrm{al}^{20}$ & \pm & - & - & - & \pm & - \\
\hline McSorley et $\mathrm{a}^{21}$ & \pm & - & - & - & - & \pm \\
\hline Moug et $\mathrm{al}^{22}$ & - & - & - & \pm & \pm & \pm \\
\hline Nakagawa et a ${ }^{23}$ & - & \pm & - & - & \pm & - \\
\hline $\mathrm{Ni}$ et al ${ }^{24}$ & - & - & \pm & - & \pm & - \\
\hline Nozoe et a ${ }^{25}$ & - & \pm & - & - & \pm & \pm \\
\hline Okimoto et $\mathrm{a}^{26}$ & - & - & - & - & \pm & - \\
\hline Okugawa et al ${ }^{27}$ & - & \pm & - & - & - & - \\
\hline Read et a ${ }^{28}$ & \pm & - & - & - & - & - \\
\hline Sharma et $\mathrm{a}^{29}$ & \pm & \pm & - & - & \pm & - \\
\hline Shibutani et al $(\mathrm{I})^{30}$ & - & \pm & - & - & \pm & - \\
\hline Shibutani et al $(2)^{30}$ & - & - & - & - & \pm & - \\
\hline Shimura et $\mathrm{al}^{31}$ & - & - & \pm & - & - & - \\
\hline Sirniö et $\mathrm{al}^{32}$ & - & - & - & - & \pm & - \\
\hline Son et $\mathrm{al}^{33}$ & - & - & - & - & \pm & - \\
\hline Song et $\mathrm{al}^{34}$ & - & - & - & - & - & \pm \\
\hline Sugimoto et $\mathrm{a}^{35}$ & \pm & - & - & - & \pm & - \\
\hline Sun et $\mathrm{al}^{36}$ & - & \pm & - & - & \pm & - \\
\hline Toiyama et $\mathrm{al}^{37}$ & - & - & - & - & - & \pm \\
\hline 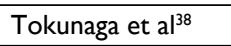 & - & - & - & - & \pm & - \\
\hline Watt et a ${ }^{39}$ & - & - & - & \pm & \pm & - \\
\hline 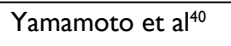 & \pm & - & - & - & \pm & \pm \\
\hline
\end{tabular}

Note: The risk of bias for each domain is graded as low $(-)$, moderate $( \pm)$, or high (+).

\section{References}

1. Adachi T, Hinoi T, Hattori M, et al. The modified Glasgow prognostic score for early mortality in patients with synchronous peritoneal carcinomatosis from colorectal cancer. Surg Today. 2015;45(11):1396-1403.

2. Chan JC, Chan DL, Diakos CI, et al. The lymphocyte-to-monocyte ratio is a superior predictor of overall survival in comparison to established biomarkers of resectable colorectal cancer. Ann Surg. 2017;265(3):539-546.
3. Choi KW, Hong SW, Chang YG, et al. Inflammation-based score (Glasgow prognostic score) as an independent prognostic factor in colorectal cancer patients. Ann Surg Treat Res. 2014;86(6):309-313.

4. Dréanic J, Maillet M, Dhooge M, et al. Prognostic value of the Glasgow Prognostic Score in metastatic colorectal cancer in the era of anti-EGFR therapies. Med Oncol. 2013;30(3):656.

5. Eren T, Burcu B, Tombalak E, et al. Clinical significance of the glasgow prognostic score for survival after colorectal cancer surgery. $J$ Gastrointest Surg. 2016;20(6):1231-1238. 
6. Furukawa $\mathrm{K}$, Shiba $\mathrm{H}$, Haruki $\mathrm{K}$, et al. The Glasgow prognostic score is valuable for colorectal cancer with both synchronous and metachronous unresectable liver metastases. Oncol Lett. 2012;4(2):324-328.

7. Ghanim B, Schweiger T, Jedamzik J, et al. Elevated inflammatory parameters and inflammation scores are associated with poor prognosis in patients undergoing pulmonary metastasectomy for colorectal cancer. Interact Cardiovasc Thorac Surg. 2015;21(5):616-623.

8. Hong T, Shen D, Chen X, Cai D, Wu X, Hua D. A novel systematic inflammation related index is prognostic in curatively resected nonmetastatic colorectal cancer. Am J Surg. 2018;216(3):450-457.

9. Ide S, Toiyama Y, Okugawa $\mathrm{Y}$, et al. Clinical significance of C-reactive protein-to-albumin ratio with rectal cancer patient undergoing chemoradiotherapy followed by surgery. Anticancer Res. 2017;37(10): 5797-5804.

10. Inoue $Y$, Iwata $T$, Okugawa $Y$, et al. Prognostic significance of a systemic inflammatory response in patients undergoing multimodality therapy for advanced colorectal cancer. Oncology. 2013;84(2):100-107.

11. Ishizuka M, Nagata H, Takagi K, Iwasaki Y, Kubota K. Inflammationbased prognostic system predicts postoperative survival of colorectal cancer patients with a normal preoperative serum level of carcinoembryonic antigen. Ann Surg Oncol. 2012;19(11):3422-3431.

12. Ishizuka M, Nagata H, Takagi K, Iwasaki Y, Shibuya N, Kubota $\mathrm{K}$. Clinical significance of the $\mathrm{C}$-reactive protein to albumin ratio for survival after surgery for colorectal cancer. Ann Surg Oncol. 2016;23(3):900-907.

13. Kim IH, Lee JE, Yang JH, Jeong JW, Ro S, Lee MA. Clinical significance of changes in systemic inflammatory markers and carcinoembryonic antigen levels in predicting metastatic colorectal cancer prognosis and chemotherapy response. Asia Pac J Clin Oncol. 2018;14(3):239-246.

14. Kishiki T, Masaki T, Matsuoka H, et al. Modified Glasgow prognostic score in patients with incurable stage IV colorectal cancer. Am J Surg. 2013;206(2):234-240.

15. Kobayashi T, Teruya M, Kishiki T, et al. Elevated C-reactive protein and hypoalbuminemia measured before resection of colorectal liver metastases predict postoperative survival. Dig Surg. 2010;27(4):285-290.

16. Køstner AH, Kersten C, Löwenmark T, et al. The prognostic role of systemic inflammation in patients undergoing resection of colorectal liver metastases: C-reactive protein (CRP) is a strong negative prognostic biomarker. J Surg Oncol. 2016;114(7):895-899.

17. Leitch EF, Chakrabarti M, Crozier JE, et al. Comparison of the prognostic value of selected markers of the systemic inflammatory response in patients with colorectal cancer. Br J Cancer. 2007;97(9):1266-1270.

18. Lin MS, Huang JX, Yu H. Prognostic significance of Glasgow prognostic score in patients with stage II colorectal cancer. Int J Clin Exp Med. 2015;8(10):19138-19143.

19. Maeda K, Shibutani M, Otani H, et al. Prognostic value of preoperative inflammation-based prognostic scores in patients with stage IV colorectal cancer who undergo palliative resection of asymptomatic primary tumors. Anticancer Res. 2013;33(12):5567-5573.

20. Maillet M, Dréanic J, Dhooge M, et al. The predictive and prognostic value of the Glasgow Prognostic Score in metastatic colorectal carcinoma patients receiving bevacizumab. Anticancer Drugs. 2014;25(10):1215-1219.

21. McSorley ST, Khor BY, Tsang K, et al. The relationship between ${ }^{18} \mathrm{~F}$-FDG-PETCT-derived markers of tumour metabolism and systemic inflammation in patients with recurrent disease following surgery for colorectal cancer. Colorectal Dis. 2018;20(5):407-415.

22. Moug SJ, McColl G, Lloyd SM, Wilson G, Saldanha JD, Diament RH. Comparison of positive lymph node ratio with an inflammation-based prognostic score in colorectal cancer. Br J Surg. 2011;98(2):282-286.
23. Nakagawa K, Tanaka K, Nojiri K, et al. The modified Glasgow prognostic score as a predictor of survival after hepatectomy for colorectal liver metastases. Ann Surg Oncol. 2014;21(5):1711-1718.

24. Ni X, Wu P, Wu J, et al. C-reactive protein/albumin ratio as a predictor of survival of metastatic colorectal cancer patients receiving chemotherapy. Int J Clin Exp Pathol. 2016;9:5525-5534.

25. Nozoe T, Matono R, Ijichi H, Ohga T, Ezaki T. Glasgow Prognostic Score (GPS) can be a useful indicator to determine prognosis of patients with colorectal carcinoma. Int Surg. 2014;99(5):512-517.

26. Okimoto S, Kobayashi T, Tashiro H, et al. Significance of the Glasgow Prognostic Score for patients with colorectal liver metastasis. Int J Surg. 2017;42:209-214.

27. Okugawa Y, Shirai Y, Toiyama Y, et al. Clinical Burden of Modified Glasgow Prognostic Scale in Colorectal Cancer. Anticancer Res. 2018;38(3):1599-1610.

28. Read JA, Choy ST, Beale PJ, Clarke SJ. Evaluation of nutritional and inflammatory status of advanced colorectal cancer patients and its correlation with survival. Nutr Cancer. 2006;55(1):78-85.

29. Sharma R, Zucknick M, London R, Kacevska M, Liddle C, Clarke SJ. Systemic inflammatory response predicts prognosis in patients with advanced-stage colorectal cancer. Clin Colorectal Cancer. 2008;7(5):331-337.

30. Shibutani M, Maeda K, Nagahara H, et al. Significance of markers of systemic inflammation for predicting survival and chemotherapeutic outcomes and monitoring tumor progression in patients with unresectable metastatic colorectal cancer. Anticancer Res. 2015;35(9):5037-5046.

31. Shimura T, Toiyama Y, Saigusa S, et al. Inflammation-based prognostic scores as indicators to select candidates for primary site resection followed by multimodal therapy among colorectal cancer patients with multiple metastases. Int J Clin Oncol. 2017;22(4):758-766.

32. Sirniö P, Tuomisto A, Tervahartiala T, et al. High-serum MMP-8 levels are associated with decreased survival and systemic inflammation in colorectal cancer. Br J Cancer. 2018;119(2):213-219.

33. Son HJ, Park JW, Chang HJ, et al. Preoperative plasma hyperfibrinogenemia is predictive of poor prognosis in patients with nonmetastatic colon cancer. Ann Surg Oncol. 2013;20(9):2908-2913.

34. Song A, Eo W, Lee S. Comparison of selected inflammation-based prognostic markers in relapsed or refractory metastatic colorectal cancer patients. World J Gastroenterol. 2015;21(43):12410-12420.

35. Sugimoto K, Komiyama H, Kojima Y, Goto M, Tomiki Y, Sakamoto K. Glasgow prognostic score as a prognostic factor in patients undergoing curative surgery for colorectal cancer. Dig Surg. 2012;29(6):503-509.

36. Sun ZQ, Han XN, Wang HJ, et al. Prognostic significance of preoperative fibrinogen in patients with colon cancer. World J Gastroenterol. 2014;20(26):8583-8591.

37. Toiyama Y, Miki C, Inoue Y, Tanaka K, Mohri Y, Kusunoki M. Evaluation of an inflammation-based prognostic score for the identification of patients requiring postoperative adjuvant chemotherapy for stage II colorectal cancer. Exp Ther Med. 2011;2(1):95-101.

38. Tokunaga R, Sakamoto Y, Nakagawa S, et al. Comparison of systemic inflammatory and nutritional scores in colorectal cancer patients who underwent potentially curative resection. Int $J$ Clin Oncol. 2017;22(4):740-748.

39. Watt DG, McSorley ST, Park JH, Horgan PG, McMillan DC. A postoperative systemic inflammation score predicts short- and long-term outcomes in patients undergoing surgery for colorectal cancer. Ann Surg Oncol. 2017;24(4):1100-1109.

40. Yamamoto M, Yoshinaga K, Matsuyama A, et al. Plasma D-dimer level as a mortality predictor in patients with advanced or recurrent colorectal cancer. Oncology. 2012;83(1):10-15. 
Cancer Management and Research

\section{Publish your work in this journal}

Cancer Management and Research is an international, peer-reviewed open access journal focusing on cancer research and the optimal use of preventative and integrated treatment interventions to achieve improved outcomes, enhanced survival and quality of life for the cancer patient.

The manuscript management system is completely online and includes

Submit your manuscript here: https://www.dovepress.com/cancer-management-and-research-journal 